\title{
Modeling the Structure and Activity of Comet Nuclei
}

\author{
Dina Prialnik \\ Tel Aviv University
}
Johannes Benkhoff
DLR Berlin

\author{
Morris Podolak \\ Tel Aviv University
}

\begin{abstract}
Numerical simulation of the structure and evolution of a comet nucleus is reviewed from both the mathematical and the physical point of view. Various mathematical procedures and approximations are discussed, and different attempts to model the physical characteristics of cometary material, such as thermal conductivity, permeability to gas flow, drag of dust grains, and dust mantling, are described. The evolution and activity of comets is shown to depend on different classes of parameters: defining parameters, such as size and orbit; structural parameters, such as porosity and composition; and initial parameters, such as temperature and live radioisotope content. Despite the large number of parameters, general conclusions or common features appear to emerge from the numerous model calculations - for different comets performed to date. Thus, the stratified structure of comet nuclei, volatile depletion, and the role of crystallization of ice in cometary outbursts are discussed.
\end{abstract}

\section{INTRODUCTION}

Although comets have been observed and studied since antiquity, the comet nucleus is a much more recent concept: "It has been stated that within the head of a comet there is usually a bright point termed the nucleus. This is the only part of its structure that excites any suspicion of a solid substance." (Robert Grant, History of Physical Astronomy, 1852). Even now, only two comet nuclei (Halley and Borrelly) have been observed at close distances, where their shapes and sizes can be clearly seen, and none has yet been probed beneath the surface. Nevertheless, it is the nucleus that, by its structure and composition, determines the behavior of a comet in a given orbit.

One of the striking features of comet nuclei is their varied, often unexpected, behavior. Some exhibit outbursts when they are close to the Sun, others when they are far from the Sun. Some show a drastic reduction in their gas outflow for several orbits, during which they have a distinctly asteroidal appearance. Some nuclei suddenly split into several smaller pieces, while others remain whole even when they pass sufficiently close to the Sun to be affected by tidal forces. In short, every comet nucleus seems to have its own special pattern of behavior. Thus it is a real challenge to develop a theory of comet nucleus behavior that is rich enough to allow for this wealth of idiosyncrasy, albeit based on a handful of relatively simple processes, such as are expected of a moderately large icy rock floating in space. Remarkably, such a theory — or model — seems to be possible and has aroused growing interest and achieved increasing sophistication during the past two or three decades. A list of symbols and constants used in the mathematical formulation of comet nucleus models is given in Table 1.

\subsection{Historical Perspective}

The simplest view of a comet nucleus is that of an active surface enveloping an inert interior. It stems from the assumption that solar radiation - exterior to the nucleus is the only energy source responsible for cometary activity. Thus the Sun's gravity determines the dynamic history of a comet and solar energy determines its thermal history. Given a composition of ice and dust, the thermal properties of cometary material appear to be such that the skin depth associated with the orbital cycle is much smaller than the radius, and hence an inert interior seems to be justified. This is also the reason for considering comets as pristine, unaltered objects, relics of the formation of the solar system. This naive view will be shown to have changed considerably in recent years. Nevertheless, the simplest among comet nucleus models deal with the surface and assume constant properties over its entire extent (albedo, emissivity, and dust/ice mass ratio), completely neglecting any energy exchange with the interior. The earliest model based on these assumptions was that of Squires and Beard (1961); later models were calculated by Cowan and A'Hearn (1979). They provided solutions for the power balance equation at the nucleus boundary to obtain the variation of surface temperatures and gas and dust production rates as a function of 
TABLE 1. List of symbols.

\begin{tabular}{ll|ll|ll}
\hline $\mathcal{A}$ & Albedo & $\mathrm{P}_{\text {orb }}$ & Orbital period & $\mathrm{v}$ & Velocity \\
$\mathrm{a}$ & Semimajor axis & $\mathrm{P}_{\text {spin }}$ & Nucleus spin period & $\mathrm{v}_{\text {th }}$ & Thermal velocity \\
$\mathrm{c}$ & Specific heat & $\mathrm{P}_{\alpha}$ & Partial gas pressure & $\mathrm{X}_{\alpha}$ & Mass fraction of species $\alpha$ \\
$\mathrm{d}_{\mathrm{H}}$ & Heliocentric distance & $\mathcal{P}_{\alpha}$ & Saturated vapor pressure & $\varepsilon$ & Emissivity \\
$\mathrm{e}$ & Eccentricity & $\dot{\mathrm{Q}}_{\mathrm{rad}}$ & Radioenergy generation rate & $\zeta$ & Angle of insolation \\
$\mathrm{F}$ & Energy flux & $\mathrm{Q}_{\alpha}$ & Surface sublimation flux & $\theta$ & Latitude \\
$f_{\alpha}$ & Fraction of trapped gas & $\mathrm{q}_{\alpha}$ & Volume sublimation rate & $\vartheta$ & Declination \\
$\mathrm{G}$ & Gravitational constant & $\mathrm{R}$ & Radius of nucleus & $\kappa$ & Thermal diffusivity \\
$\mathrm{g}$ & Gravitational acceleration & $\mathcal{R}_{\mathrm{g}}$ & Ideal gas constant & $\lambda$ & Crystallization rate \\
$\mathcal{H}_{\mathrm{ac}}$ & Heat of crystallization & $\mathrm{r}$ & Radial distance from center & $\mu$ & Molecular weight \\
$\mathcal{H}_{\alpha}$ & Latent heat of sublimation & $\mathrm{r}_{\mathrm{d}}$ & Dust grain radius & $\mathrm{v}$ & Kinematic viscosity \\
$\mathrm{J}$ & Mass flux & $\mathrm{r}_{\mathrm{d}}^{*}$ & Critical dust grain radius & $\xi$ & Tortuosity \\
$\mathrm{K}$ & Thermal conductivity & $\mathrm{r}_{\mathrm{p}}$ & Pore radius & $\rho_{\alpha}$ & Partial density of ice species \\
$\mathrm{k}$ & Boltzmann constant & $\mathrm{S}$ & Surface to volume ratio & $\tilde{\rho}_{\alpha}$ & Partial density of gas species \\
$\mathrm{L}_{\odot}$ & Solar luminosity & $\mathrm{T}$ & Temperature & $\varrho_{\alpha}$ & Density of solid species \\
$\ell$ & Mean free path & $\mathcal{T}$ & Tensile strength & $\sigma$ & Stefan-Boltzmann constant \\
$\mathrm{M}$ & Mass of nucleus & $\mathrm{t}$ & Time & $\tau$ & Characteristic timescale \\
$\mathrm{M}_{\odot}$ & Solar mass & $\mathrm{u}$ & Energy per unit mass & $\Psi$ & Porosity \\
$\mathrm{m}_{\alpha}$ & Molecular mass of species $\alpha$ & $\mathrm{V}$ & Volume & $\omega$ & Hour angle \\
\hline
\end{tabular}

heliocentric distance. A so-called "standard model" emerged, based on power balance for a unit area normal to the solar direction

$$
\frac{(1-\mathcal{A}) \mathrm{L}_{\odot}}{4 \pi \mathrm{d}_{\mathrm{H}}^{2}}=\varepsilon \sigma \mathrm{T}^{4}+\mathcal{P}_{\mathrm{H}_{2} \mathrm{O}} \sqrt{\mathrm{m}_{\mathrm{H}_{2} \mathrm{O}} / 2 \pi \mathrm{kT}} \mathcal{H}_{\mathrm{H}_{2} \mathrm{O}}
$$

assuming the surface to be entirely covered by water ice. The total incident energy depends on the cross-sectional area of the nucleus, whereas evaporation and reradiation of energy occur over the entire hemispherical surface facing the Sun. However, since regions not normal to the incident radiation receive less energy by a cosine factor and are at a lower temperature, less energy is lost from these regions by evaporation and radiation. Therefore, in view of the great uncertainties in the physical properties of cometary material, the comet was treated as a two-dimensional (2-D) disk of area $\pi \mathrm{R}^{2}$ facing the Sun. Combined with observed production rates, this model is often used in order to estimate the nucleus size (or at least the size of its active surface area).

Diurnal temperature variations over the surface of the nucleus were first studied by Weissman and Kieffer (1981, 1984) and by Fanale and Salvail (1984). Heat conduction to the interior was now considered as well, but only in a thin subsurface layer. The possibility of an outer dust mantle enveloping the icy nucleus was first considered by Mendis and Brin (1977) and Brin and Mendis (1979), and subsequently pursued by many others. Heat conduction throughout the entire nucleus was first explored by Herman and Podolak (1985), who solved the heat diffusion equation for the one-dimensional (1-D) case of a homogeneous spherical nucleus. It was prompted by the suggestion that cometary ice could be amorphous and its subsequent crystallization could provide an internal source of energy (Patashnik et al., 1974), so far ignored. This study explored the consequences of crystallization of amorphous ice and the associated release of latent heat on the temperature profile. It revealed intermittent bursts of crystallization, an effect that was studied in considerably more detail by Prialnik and Bar-Nun (1987), reviving an earlier suggestion that cometary outbursts might be linked with the crystallization process. In parallel, cometary activity due to volatiles other than $\mathrm{H}_{2} \mathrm{O}$ was studied by Fanale and Salvail (1987).

The next step in the study of comet nuclei by numerical simulations was prompted by the detailed observations of Comet P/Halley when it passed perihelion in 1986, which revealed that the nucleus had a very low bulk density, indicating a high porosity (Rickman, 1989). Further evidence in favor of porosity is provided by the presence in cometary ejecta of molecules from volatile ices, which cannot survive in the warm subsurface layers. The origin of such molecules must therefore be in the deeper, colder layers of the nucleus. Pores act as conduits for the transport of gases trapped in comet nuclei. Mekler et al. (1990) initiated a detailed study of the effect of porosity on the cometary nucleus. They developed a model of gas flow through a porous medium, allowing for vaporization from the pore walls, and used this model for the simplest case of a porous pure-water-ice nucleus. They found that for a given distance from the Sun there is a critical depth above which the gas flows out of the nucleus, and below which it flows toward the center. Laboratory experiments performed by the KOSI group around the same time (Spohn et al., 1989) confirmed these findings and promoted the study of gas flow through porous comet nuclei. The basic model of lowdensity flow through a porous medium has been adapted by a number of research groups (e.g., Espinasse et al., 1991; Steiner and Kömle, 1991; Prialnik, 1992; Tancredi et al., 1994; Benkhoff and Huebner, 1995). More recently, at- 
tempts have been made to include the flow of dust particles through the pores as well (Orosei et al., 1995; Podolak and Prialnik, 1996). It is now commonly agreed that porosity must be included in order to properly understand cometary behavior. A different question that has been repeatedly addressed concerns the end state of comets. Unless they are disrupted by tidal forces or destroyed by collisions with larger bodies, comet nuclei are expected to evaporate and disintegrate, leaving behind a trail of debris. But they may also become extinct - asteroid-like - if a dust mantle forms at the surface, quenching all types of cometary activity. This line of investigation started with the early work of Shul'man (1972), followed by numerous studies of various aspects of the dust mantle, culminating in detailed evolutionary models of transition objects between comets and asteroids (e.g., Coradini et al., 1997a,b).

As the prevailing idea of pristine comet nuclei interiors began to give way to more elaborate pictures of these objects, another internal heat source — radioactive decay, commonly considered for large bodies of the solar system - came into focus. The first to have considered radioactive heating of comet nuclei were Whipple and Stefanik (1966): They found that the decay of ${ }^{40} \mathrm{~K},{ }^{235} \mathrm{U},{ }^{238} \mathrm{U}$, and ${ }^{232} \mathrm{Th}$ caused the internal temperature to rise to a peak of $\sim 90 \mathrm{~K}$ (from an initial $0 \mathrm{~K}$ ) on a timescale of some $10^{8} \mathrm{yr}$. About 10 years later, Lee et al. (1976) presented strong evidence that the short-lived radionuclide ${ }^{26} \mathrm{Al}$ had been present in the early solar nebula. Further evidence strengthening this conclusion has accumulated ever since. The idea of internal heating of comet nuclei by the decay of ${ }^{26} \mathrm{Al}$ gained impetus following early studies by Irvine et al. (1980) and Wallis (1980), who showed that it may lead to melting of the ice, which would have implications for early formation of organic molecules and the origin of life (see Thomas et al., 1997). Thus heating by radioactive decay has been considered in a number of comet nucleus models under various conditions and assumptions. Whether or not liquid water could have been present in comet nuclei during their early stages of evolution, and if so, under which conditions, is still debated. The question of whether and to what extent comets are pristine bodies that hold clues to the formation of the solar system is still open. Finally, would it be possible for comet nuclei to have had liquid cores, but at the same time preserved their outer layers in pristine form? These are some of the questions that have prompted the development of increasingly sophisticated models of comet nuclei. The number of studies devoted to the evolution and activity of comet nuclei and to the complex processes involved is steadily growing. The effort is twofold: understanding and providing a usable mathematical formulation of the processes on the one hand, and incorporating these processes in numerical simulations of the structure and evolution of comet nuclei on the other. Section 3 will be devoted to the former and section 4 to the latter. The set of evolutionary equations will be presented in section 2 , and conclusions as well as suggestions for future work, in section 5.

\subsection{Basic Assumptions Derived from Observations}

The structure of a comet nucleus may be modeled as a highly porous agglomeration of grains made of volatile ices and dust, with a size distribution that probably spans many orders of magnitude. The dominant volatile component is water ice, while the other volatiles, such as $\mathrm{CO}, \mathrm{CO}_{2}, \mathrm{HCN}$, $\mathrm{N}_{2}$, etc., are mixed with the water ice or incorporated in it, either in the form of clathrate-hydrates, or as trapped gases within the (amorphous) ice matrix. Having been formed at low temperatures and pressures, cometary ice is believed to be amorphous (Mekler and Podolak, 1994). Laboratory experiments indicate that amorphous ice is capable of trapping large amounts of gas and most of this trapped gas escapes when the ice crystallizes (Bar-Nun et al., 1987). Thus, whereas $\mathrm{H}_{2} \mathrm{O}$ molecules are released within a narrow temperature range, when the ice sublimates, the other volatiles may be released in different temperature ranges. Crystallization, as well as sublimation from the pore walls in the deep cometary interior, may be triggered (and sustained) either by a heat wave advancing inward from the surface, or due to internal heat release by radioactive isotopes contained in the dust, or else by the release of latent heat that accompanies the transformation of amorphous into crystalline ice.

These are, in fact, the three main - and perhaps only sources of energy available to comets. Their typical properties are summarized in Table 2 . The radioactive source is important particularly during the long period of time spent by comets outside the planetary system, far from the Sun. Close to the Sun, it is far less efficient than solar radiation, and hence negligible. The most important radionuclide is the short-lived isotope ${ }^{26} \mathrm{Al}$. Observational evidence points toward an interstellar isotopic ratio ${ }^{26} \mathrm{Al} /{ }^{27} \mathrm{Al} \approx 5 \times 10^{-5}$ (e.g., MacPherson et al., 1995), implying an initial mass fraction $\mathrm{X}_{0}\left({ }^{26} \mathrm{Al}\right) \approx 7 \times 10^{-7}$ in the solar nebula dust (rock) and presumably an order of magnitude less, on average, in objects such as comets, for which the time of aggregation did not exceed a few million years (Lugmair and Shukolyukov, 2001). In contrast to these sources, the exoergic crystallization of amorphous water ice is not an independent source, since it occurs above a threshold temperature that must be attained by means of other energy sources. Crystallization may occur at any evolutionary stage, and may propagate either inward or outward.

Once gas is released from the ice in the interior of the nucleus, its pressure will cause it to flow to the surface. Gases moving through the pores drag with them small dust

TABLE 2. Energy sources and their characteristics.

\begin{tabular}{ccc}
\hline Solar Radiation & Radioactivity & Crystallization \\
\hline Surface source & Body source & Local source \\
$\propto \mathrm{R}^{2} / \mathrm{d}_{\mathrm{H}}^{2}$ & $\propto \mathrm{R}^{3} \mathrm{X}_{\mathrm{rad}}$ & $\propto \mathrm{X}_{\mathrm{a}-\mathrm{ice}} \mathcal{H}_{\mathrm{ac}}$ \\
Cyclic & Declining & Transient \\
Inward moving wave & Homogeneous & Thin front \\
Late evolution & Early evolution & Induced \\
\hline
\end{tabular}


particles that have detached from the solid matrix. The larger particles may eventually block the pores; the smaller ones may flow all the way with the gas.

The free gases present in the interior of a comet are expected to affect the thermal and mechanical structure of the nucleus by contributing to the conduction of heat through advection or recondensation and by building up internal pressure. This pressure may surpass the tensile strength of the already fragile, grainy configuration and result in cracking of the porous matrix and outbursts of gas and dust. Accumulation of large particles on the nucleus surface may lead to the formation of a sealing dust mantle that may partially (or fully) quench the comet's activity.

All these processes are taken into account in models of the evolution and activity of comet nuclei, as will be shown in the next section. Internal processes depend on physical properties characteristic of cometary material, which will be discussed in sections 3 and 4 .

\subsection{Approximations Required by Modeling}

Comet nuclei are too small for self-gravity to be of importance, hence they are not necessarily spherical. However, a nonspherical object is far more difficult to model. In addition, the number of free parameters for an arbitrary shape tends to infinity. Thus models must assume some form of symmetry and sphericity, requiring a single dimensional parameter - the (effective) radius $\mathrm{R}$ is the common assumption. The simplest among spherical models are 1-D, considering a spherically symmetric nucleus, which implies an evenly heated surface, although in reality only one hemisphere faces the Sun at any given time, and even its surface is not evenly irradiated. This approximation is known (somewhat loosely) as the "fast-rotator" approximation. It is a good approximation for the interior of the nucleus, below the skin depth; it is valid for the surface far away from the Sun, where diurnal temperature variations are small.

However, in order to obtain an accurate surface temperature distribution and its diurnal change at any heliocentric distance, one must adopt the so-called "slow-rotator" approach, which takes into account the diurnal and latitudinal solar flux variations. This type of model requires a far greater amount of computing time, since much smaller time steps - a small fraction of the spin period - must be used in the numerical integration over time.

A first attempt in this direction was to consider a point on the equator of a spinning nucleus and translate the diurnal temperature change obtained into a map of the equatorial temperature at any given time. Such a procedure (Benkhoff and Boice, 1996; Benkhoff, 1999) may be described as a 1.5-dimensional (1.5-D) model. An upper limit for the production rate is obtained by using the maximum noon flux for the entire surface of the sunlit hemisphere. A more advanced model is achieved by considering a wedge of surface elements aligned along a meridian (Enzian et al., 1997, 1999). Thus the latitudinal effect is taken into account and the total production rate is obtained by summing the con-

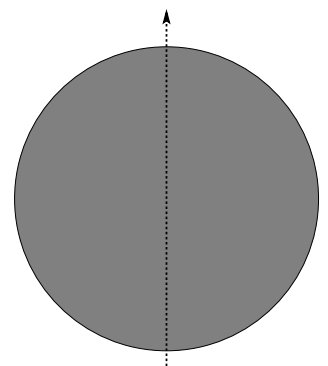

1-D "fast rotator"

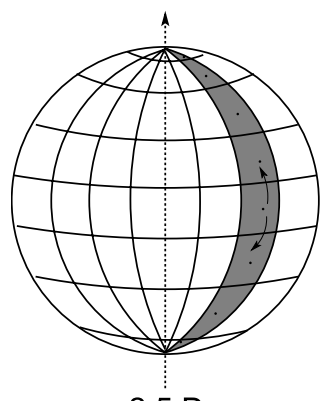

$2.5 \mathrm{D}$
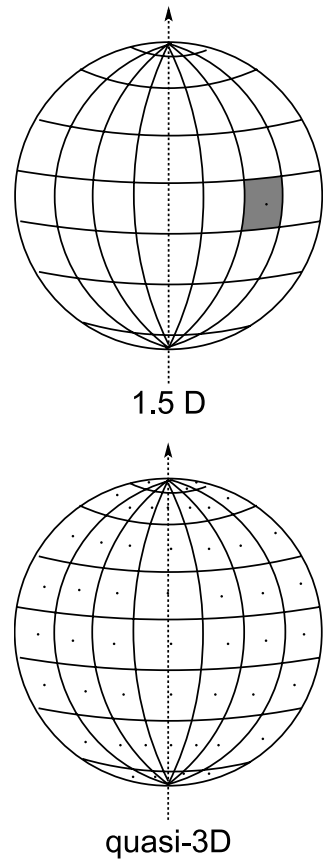

Fig. 1. Schematical representation of numerical grids for a spinning nucleus, commonly used in model calculations. Dots indicate radial directions along which heat conduction is computed; lateral conduction is only included in the $2.5-\mathrm{D}$ model, and only along the meridian, as shown.

tributions of such wedges over one spin period. This is essentially a 2.5 -dimensional (2.5-D) calculation. The next step is to take into account both diurnal and latitudinal solar flux variations (Gutiérrez et al., 2000; Julian et al., 2000; Cohen et al., 2003), considering, however, only radial heat conduction, i.e., neglecting lateral conduction. This quasi three-dimensional (3-D) approach is amply justified by the extremely low heat conductivity of cometary material; the characteristic heat diffusion time between the equator and pole (as between surface and center) is of the order of the lifetime of a comet (see Table 5 below). The different models are shown schematically in Fig. 1; of course, calculations use much finer meshes than the ones shown.

The advantage of the negligible self-gravity of comets is that the structure of the nucleus may be assumed incompressible despite the low strength of the porous material. By comparing the hydrostatic pressure at the center with the material strength, we obtain the condition for incompressibility

$$
\rho \mathrm{R} \leq \sqrt{3 C / 2 \pi \mathrm{G}}
$$

where $C$ is the compressive strength. Even for as low a value as $C \approx 10 \mathrm{kPa}$ (see section 3.8), this implies

$$
\rho_{\mathrm{g} / \mathrm{cm} 3} R_{\mathrm{km}} \leq 10
$$

which is amply satisfied by the typical sizes and densities 
of comet nuclei. Therefore, the equations that determine the structure and evolution of comets are those of energy conservation and of mass conservation for the various components. Momentum conservation (hydrostatic balance) is not required for the solid matrix and can be replaced by a prescribed (usually constant) density profile; for the gas species, expressions for the flux in different regimes are used.

\section{EVOLUTION EQUATIONS AND SOLUTION METHODS}

\subsection{Mass and Energy Conservation}

Consider a composition of water ice and vapor, dust, and other volatiles, which, as we have seen, may be frozen, free, or trapped in the amorphous water ice. For each volatile species $\alpha$ we distinguish — when necessary — between two phases, solid (denoted by index s) and gas (index g), which have, e.g., different specific heat coefficients. For water, the solid phase may be either amorphous (index a) or crystalline (index c); water vapor will be denoted by index $\mathrm{v}$ and dust by $\mathrm{d}$. Then the mass density and porosity are given respectively by

$$
\begin{gathered}
\rho=\rho_{\mathrm{a}}+\rho_{\mathrm{c}}+\rho_{\mathrm{v}}+\sum_{\alpha}\left(\rho_{\mathrm{s}, \alpha}+\rho_{\mathrm{g}, \alpha}\right)+\rho_{\mathrm{d}} \\
\Psi=1-\left(\rho_{\mathrm{a}}+\rho_{\mathrm{c}}\right) / \varrho_{\mathrm{H}_{2} \mathrm{O}}-\sum_{\alpha} \rho_{\mathrm{s}, \alpha} / \varrho_{\alpha}-\rho_{\mathrm{d}} / \varrho_{\mathrm{d}}
\end{gathered}
$$

We note that densities refer to mass per unit volume of nucleus material. Since the gas resides in the pores, the actual gas density within the pores will be $\rho_{\mathrm{g}, \alpha} / \Psi$ and the partial pressure, assuming an ideal gas, will be

$$
\mathrm{P}_{\alpha}=\frac{\mathcal{R}_{\mathrm{g}} \rho_{\mathrm{g}, \alpha} \mathrm{T}}{\Psi \mu_{\alpha}}
$$

Local thermodynamic equilibrium is assumed to prevail, that is, all components in all phases, as well as radiation, share the same local temperature. It is further assumed that gases trapped in the amorphous ice do not affect its heat capacity or density. Let $f$ be the total fraction of occluded gas, $\Sigma_{\alpha} f_{\alpha}=f$. Thus the equations of mass conservation are

$$
\begin{gathered}
\frac{\partial \rho_{\mathrm{a}}}{\partial \mathrm{t}}=-\lambda \rho_{\mathrm{a}} \\
\frac{\partial \rho_{\mathrm{c}}}{\partial \mathrm{t}}=(1-f) \lambda \rho_{\mathrm{a}}-\mathrm{q}_{\mathrm{v}} \\
\frac{\partial \rho_{\mathrm{v}}}{\partial \mathrm{t}}+\nabla \cdot \mathbf{J}_{\mathrm{v}}=\mathrm{q}_{\mathrm{v}}
\end{gathered}
$$

for $\mathrm{H}_{2} \mathrm{O}$ in all its phases, and similarly

$$
\begin{aligned}
\frac{\partial \rho_{\mathrm{g}, \alpha}}{\partial \mathrm{t}}+\nabla \cdot \mathbf{J}_{\alpha} & =f_{\alpha} \lambda \rho_{\mathrm{a}}+\mathrm{q}_{\alpha} \\
\frac{\partial \rho_{\mathrm{s}, \alpha}}{\partial \mathrm{t}} & =-\mathrm{q}_{\alpha}
\end{aligned}
$$

The equation of energy conservation is

$$
\frac{\partial}{\partial \mathrm{t}}[\rho \mathrm{u}]+\nabla \cdot\left(\mathbf{F}+\sum_{\alpha} \mathrm{u}_{\mathrm{g}, \alpha} \mathbf{J}_{\alpha}\right)=\mathcal{S}
$$

where $S$ stands for all the available energy sources and sinks,

$$
S=\lambda \rho_{\mathrm{a}} \mathcal{H}_{\mathrm{ac}}+\dot{\mathrm{Q}}_{\mathrm{rad}}-\sum_{\alpha} \mathrm{q}_{\alpha} \mathcal{H}_{\alpha}
$$

Here $[\rho u]$ represents the sum over all species and all phases (although the contribution of the gas phases is small and hence sometimes neglected), $\mathrm{u}=\int \mathrm{c}(\mathrm{T}) \mathrm{dT}$, and

$$
\mathbf{F}=-\mathrm{K} \nabla \mathrm{T}
$$

Combining the energy and mass conservation equations, we obtain the heat transfer equation, which can replace equation (10)

$$
\sum_{\alpha} \rho_{\alpha} \frac{\partial \mathrm{u}_{\alpha}}{\partial \mathrm{t}}-\nabla \cdot(\mathrm{K} \nabla \mathrm{T})+\left(\sum_{\alpha} \mathrm{c}_{\alpha} \mathbf{J}_{\alpha}\right) \cdot \nabla \mathrm{T}=\mathcal{S}
$$

with the advantage being that the temporal derivatives of the variables are now separated. The above set of time-dependent equations is subject to constitutive relations $\mathrm{u}(\mathrm{T}), \lambda(\mathrm{T})$, $\mathrm{q}_{\alpha}\left(\mathrm{T}, \Psi, \mathrm{r}_{\mathrm{p}}\right), \mathbf{J}_{\alpha}\left(\mathrm{T}, \Psi, \mathrm{r}_{\mathrm{p}}\right)$, and $\mathrm{K}\left(\mathrm{T}, \Psi, \mathrm{r}_{\mathrm{p}}\right)$, which require additional assumptions for modeling the structure of the nucleus. They will be discussed in some detail in the next section. The most widely used expressions or values for the thermal properties of cometary $\mathrm{H}_{2} \mathrm{O}$ ice and dust are given in Table 3. Ice properties have been measured and turned into empirical temperature-dependent relations by Klinger (1980, 1981), and more recently by Ross and Kargel (1998).

TABLE 3. Thermal properties of cometary $\mathrm{H}_{2} \mathrm{O}$ ice and dust.

\begin{tabular}{lcc}
\hline Property & Relation & Units \\
\hline Specific heat: $\mathrm{c}_{\mathrm{a}}, \mathrm{c}_{\mathrm{c}}$ & $7.49 \mathrm{~T}+90$ & $\mathrm{~J} \mathrm{~kg}^{-1} \mathrm{~K}^{-1}$ \\
Specific heat: $\mathrm{c}_{\mathrm{v}}=3 \mathcal{R}_{\mathrm{g}} \mu$ & $1.385 \times 10^{3}$ & $\mathrm{~J} \mathrm{~kg}^{-1} \mathrm{~K}^{-1}$ \\
Specific heat: $\mathrm{c}_{\mathrm{d}}$ & $\sim 800$ & $\mathrm{~J} \mathrm{~kg}^{-1} \mathrm{~K}^{-1}$ \\
Thermal conductivity: $\mathrm{K}_{\mathrm{c}}$ & $567 / \mathrm{T}$ & $\mathrm{J} \mathrm{m}^{-1} \mathrm{~s}^{-1} \mathrm{~K}^{-1}$ \\
Thermal diffusivity: $\kappa_{\mathrm{a}}$ & $3.13 \times 10^{-7}$ & $\mathrm{~m}^{2} \mathrm{~s}^{-1}$ \\
Thermal conductivity: $\mathrm{K}_{\mathrm{d}}$ & $\sim 0.1-4$ & $\mathrm{~J} \mathrm{~m}^{-1} \mathrm{~s}^{-1} \mathrm{~K}^{-1}$ \\
\hline
\end{tabular}




\subsection{Boundary Conditions}

The set of evolution equations must be supplemented by initial and boundary conditions. For the heat transfer equation, the boundary conditions refer to the flux $\mathrm{F}(\mathrm{r})$ on the open interval $r_{0}<r<R$, where $r_{0}=0$ when the entire comet is considered, or $0<r_{0}<R$, when only an outer layer is considered in a plane-parallel calculation. At the ends of this interval we have

$$
\begin{gathered}
\mathrm{F}\left(\mathrm{r}_{0}\right)=0 \\
\mathrm{~F}(\mathrm{R})=\varepsilon \sigma \mathrm{T}(\mathrm{R}, \mathrm{t})^{4}+ \\
\mathcal{F} \mathrm{Q} \mathcal{H}-(1-\mathcal{A}) \frac{\mathrm{L}_{\odot}}{4 \pi \mathrm{d}_{\mathrm{H}}(\mathrm{t})^{2}} \cos \xi
\end{gathered}
$$

The local solar zenith angle is given by

$$
\cos \xi=\cos \theta \cos \omega \cos \delta+\sin \theta \sin \delta
$$

where $\delta$ is the declination (see also Sekanina, 1979; Fanale and Salvail, 1984). The factor $\mathcal{F} \leq 1$ represents the fractional area of exposed ice, since the surface material is a mixture of ice and dust (Crifo and Rodionov, 1997)

$$
\mathcal{F}=\left(1+\frac{\varrho_{\text {ice }}}{\rho_{\text {ice }}} \frac{\rho_{\mathrm{d}}}{\varrho_{\mathrm{d}}}\right)^{-1}
$$

The function $\mathrm{d}_{\mathrm{H}}(\mathrm{t})$ is given in terms of the changing eccentric anomaly $\mathrm{E}$ by the familiar celestial mechanics equations

$$
\begin{gathered}
\mathrm{t}=\sqrt{\mathrm{a}^{3 / 6 M_{\odot}}}(E-e \sin \mathrm{E}) \\
d_{\mathrm{H}}=\mathrm{a}(1-\mathrm{e} \cos \mathrm{E})
\end{gathered}
$$

Similarly to the heat flux, the mass (gas) fluxes vanish at $\mathrm{r}_{0}$. At the surface $\mathrm{R}$ the gas pressures are those exerted by the coma; in the lowest approximation they may be assumed to vanish: $\mathrm{P}_{\alpha}(\mathrm{R}, \mathrm{t})=0$. [For a more elaborate discussion concerning the boundary conditions to be assumed for the gas pressure at the surface and their effect, see Crifo et al. (2004).] We should note that when the entire comet is considered, mass and heat fluxes must vanish at the center. However, at the lower boundary of a finite layer, other conditions may equally be imposed (for example, a fixed temperature and corresponding vapor pressures), but only by adopting vanishing fluxes are energy and mass conservation secured.

In a porous medium the surface is not well defined and a surface layer of finite (rather than vanishing) thickness supplies the outflowing vapor. Mekler et al. (1990) have shown that the surface layer where most of the vapor is generated is considerably thinner than the layer of ice that is lost by a comet during a perihelion passage. We are thus faced with two vastly different length scales, which imply different timescales as well. On the evolutionary timescale of the comet the thin boundary layer may be assumed to be in (quasi) steady-state. Its ice may be assumed to have crystallized. The gas fluxes from the interior may be taken as constant and their contribution to heat conduction may be neglected. In addition, plane-parallel geometry is justified in this case and hence the equations that have to be solved near the surface as a function of depth $\mathrm{z}$ are

$$
\begin{gathered}
\mathrm{dJ}_{\mathrm{v}}\left(\mathrm{T}, \mathrm{P}_{\mathrm{v}}\right) / \mathrm{dz}=\mathrm{q}_{\mathrm{v}}\left(\mathrm{T}, \mathrm{P}_{\mathrm{v}}\right) \\
\mathrm{dF}(\mathrm{T}) / \mathrm{dz}=-\mathrm{q}_{\mathrm{v}}\left(\mathrm{T}, \mathrm{P}_{\mathrm{v}}\right) \mathcal{H}
\end{gathered}
$$

The boundary conditions for this layer are equation (15) at $\mathrm{R}$ and given temperature at the lower boundary, where it is fitted to the rest of the comet. This procedure, suggested by Prialnik (1992) was also employed by Tancredi et al. (1994).

An alternative approach to the macroscopic equations of gas diffusion in a porous volatile medium is a kinetic model, which provides gas fluxes as well as gas production and loss rates, for a given temperature distribution (Skorov and Rickman, 1995; Skorov et al., 2001). It is particularly suited to the surface layer of the nucleus, near the pore openings. In this case the gas pressure at the surface is no longer required as a boundary condition, but rather it results from the calculation.

The initial conditions must be guessed, and since the comet nucleus as a whole never reaches steady-state below a skin depth of the order of meters to several tens of meters, these conditions play a significant role. This explains the importance attached to the early evolution of comets at large distances from the Sun, which determines the interior configuration of comet nuclei when they enter the inner planetary system and become active.

\subsection{Numerical Schemes}

The system of nonlinear, time-dependent, second-order partial differential equations (6)-(9) and (10) or (13) is turned into a set of difference equations that are solved numerically. They constitute a two-boundary value problem that requires relaxation methods for its solution. Let the time and space domains be divided into finite intervals $\delta \mathrm{t}_{\mathrm{n}}=\mathrm{t}_{\mathrm{n}}-\mathrm{t}_{\mathrm{n}-1}$ and $\Delta \mathrm{r}_{\mathrm{i}}=\mathrm{r}_{\mathrm{i}}-\mathrm{r}_{\mathrm{i}-1}(0 \leq \mathrm{i} \leq \mathrm{I})$, such that $\mathrm{t}_{0}=$ $0, r_{0}=0$, and $r_{i}=R$. The solution for the change of the temperature profile will be represented by a series of stepped functions $T_{i}^{n}$, where $T_{i}$ is the temperature within the interval $\Delta r_{i}$. For the simple (linear) case of heat transfer with constant coefficients, there are several possibilities for combining the space and time derivatives into a difference equation for the transport equation, among which the most common are the explicit scheme, which can be solved directly; the fully implicit scheme, which, upon rearranging terms, results in a system of I linear equations requiring the in- 
version of a tridiagonal matrix for its solution; and the Crank-Nicholson scheme, which is a modified implicit form that requires the inversion of a tridiagonal matrix as well. The explicit scheme has the disadvantage that time-steps are restricted by the Courant-Friedrichs-Levy condition, $\delta \mathrm{t} \leq$ $(\Delta \mathrm{r})^{2} / 2 \mathrm{~K}$, for a given space discretization; thus time-steps may become prohibitively small when a fine mesh is required in order to resolve sharp temperature gradients. The implicit schemes, on the other hand, are unconditionally stable for all values of the time-step. However, they require a far greater amount of computations for each time-step, prohibitively large in the case of a large spatial grid, or in the 2- or 3-D cases. The Crank-Nicholson scheme has the advantage of being second-order accurate in time, whereas the fully implicit one, as the explicit scheme, is only firstorder accurate in time. The fully implicit scheme, on the other hand, is best suited for stiff equations, i.e., when there are two or more very different timescales on which the temperature is changing (as is the case in comets). The reason is that the implicit scheme converges to the steady-state solution for large time-steps.

The same methods apply to the more complicated case when the heat capacity and thermal conductivity are functions of the temperature, and there is also a temperaturedependent source term (such as equation (13)). In this case, the difference equations of the implicit schemes must be linearized and solved iteratively. Another numerical method of solution of the nonlinear heat equation is the predictorcorrector method, essentially a two-step iterative procedure. Each iteration (or step) requires the inversion of a tridiagonal matrix. A comparison of different algorithms that were used to compute the evolution of a comet nucleus model for the same set of physical parameters is shown in Fig. 2. Each of the numerical methods mentioned above was adopted in one or the other of these algorithms, which also differ in other numerical parameters (for details, see Huebner et al., 1999). The results are remarkably similar; the agreement at low perihelion distances is excellent, but this is expected since most of the solar radiation is spent in sublimation. Differences arise at larger distances and provide an estimate of the error-bars expected from model calculations, which are otherwise difficult to assess.

Although $\frac{\partial \mathrm{u}}{\partial \mathrm{t}}=\mathrm{c}(\mathrm{T}) \frac{\partial \mathrm{T}}{\partial \mathrm{t}}$, the time difference should be taken for the energy, rather than for the temperature, in order to ensure energy conservation in the numerical scheme. Finally, in the case of a 1-D spherical coordinate system, it is convenient to choose the volume $\mathrm{V}$ enclosed within a sphere of radius $r$ for the space variable, rather than $r$, for then the equation retains the form of the plane-parallel one. From the physical point of view, it would be even better to adopt the mass enclosed within a sphere of radius $r$ as space variable, but if the mass is allowed to change during evolution as a result of internal sublimation and gas flow, the volume is a better choice. In this case the flux through $r$ must be replaced by the energy crossing the spherical surface of radius $r$ per unit time. An additional advantage of this proce-
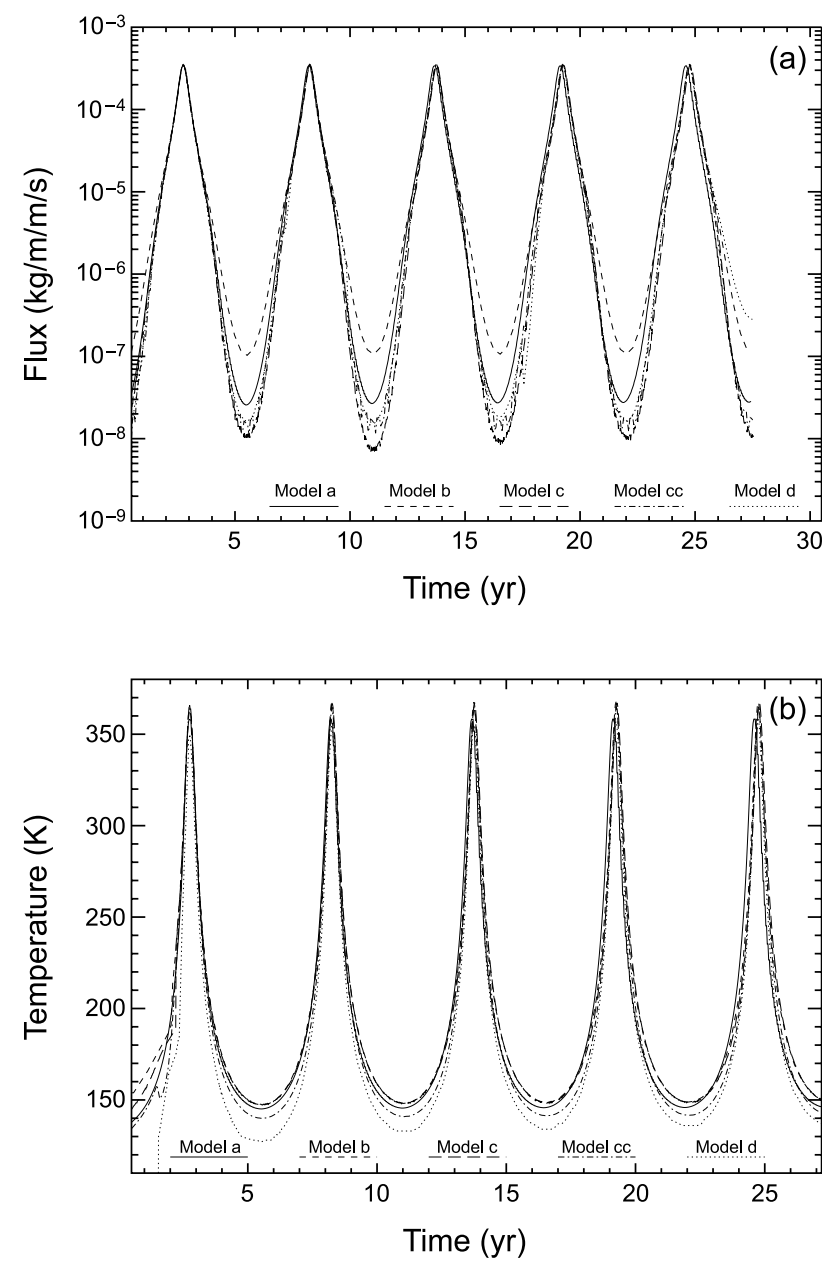

Fig. 2. Model results for (a) $\mathrm{H}_{2} \mathrm{O}$ production rates of a pure ice nucleus and (b) surface temperature of a dust-mantled nucleus, at the subsolar point as function of time for five revolutions in Comet P/Wirtanen's orbit as computed by five different algorithms (W. F. Huebner et al., personal communication, 2002).

dure is that equal volume intervals ensure a better radial resolution near the nucleus surface, where $\partial \mathrm{T} / \partial \mathrm{r}$ is steeper. Similarly, choosing the eccentric (or true) anomaly angle as the temporal variable naturally leads to smaller time steps near perihelion, where changes are more rapid.

\subsection{Computational Approximations}

We note that the evolution equations are coupled through the source terms and the gas fluxes, which are functions of both temperature and pressure, and hence must be solved simultaneously. This is extremely time consuming, considering that the equations are strongly nonlinear. Simplifying approximations may be used under special conditions.

If the effective permeability of the medium is sufficiently high, the time derivative on the lefthand side of the mass conservation equation for the gas phases (equation (8)) becomes negligible. Neglecting it is tantamount to a quasi- 
steady-state approximation, where gas densities and production rates change only as far as the temperature distribution changes. Thus equations (9) are replaced by

$$
\begin{gathered}
\nabla \cdot \mathbf{J}_{\alpha}=q_{\alpha} \\
\frac{\partial \rho_{s, \alpha}}{\partial t}=-q_{\alpha}
\end{gathered}
$$

In this way we strictly have to solve only one time-dependent equation supplemented by structure (space-dependent) equations. This constitutes a huge computational advantage, particularly in a long-term evolutionary calculation, where a detailed account of gas flow through the porous medium, coupled with heat transfer, would require a prohibitively large amount of computing time. Combining equations (22) and integrating over volume, we obtain

$$
-\dot{\mathrm{M}}_{\mathrm{s}, \alpha}=\mathrm{J}_{\alpha}(\mathrm{R}, \mathrm{t}) 4 \pi \mathrm{R}^{2}
$$

which means that the total mass of gas ejected through the comet's surface per unit time is equal to the total amount of gas evaporated throughout the nucleus per unit time for each species. This approximation is valid for nonabundant species, for which the bulk density is low. It breaks down when the net gas sublimation rate is negative, i.e., when recondensation surpasses sublimation. This approach has been recently adopted by Choi et al. (2002) in long-term evolutionary calculations of Kuiper belt objects. It is also applied for the outermost layer of the nucleus, as already mentioned in section 2.2.

A different approximation with the same computational advantage - reduction of the number of time-dependent equations - has been used in other studies (e.g., Coradini et al., 1997a) for the nucleus interior. It assumes that when both the ice and gas phases are present, the gas density is equal to the saturated vapor density, which is a function of temperature. Strictly, this would imply that no evaporation/ condensation could take place. However, as the temperature changes, the saturated density (pressure) changes with it, and this change can be translated into a rate of evaporation/ condensation. This is an excellent approximation for the interior of the nucleus, where the pressures are indeed found to attain saturation; it implies, however, that there is sufficient material in both phases to allow instantaneous adjustment. It is not valid, therefore, for minor volatile components and fails close to the surface of the nucleus. The two simplifying approximations are thus complementary.

\section{PHYSICAL PROCESSES}

\subsection{Heat Conduction in a Porous Medium}

Cometary $\mathrm{H}_{2} \mathrm{O}$ ice can be viewed as the matrix that comprises the bulk of the nucleus, but within this matrix there are grains of dust, occluded gases, and pores. Reach et al. (2000) have recently suggested that the grains dominate and form the background matrix with ice as the filler, but in either case the problem of calculating the conductivity through such a porous material remains essentially the same. For the canonical model of a porous ice matrix, the pores can themselves be filled, at different times, with smaller grains, $\mathrm{H}_{2} \mathrm{O}$ vapor, or other gases. The grains embedded in the water-ice matrix, the grains and gases flowing through the pores, and even radiation passing through the pores will each affect the rate of heat transport through the nucleus. This is a complex problem that has a rather long history.

To begin, let us consider a simple idealized medium consisting of bulk $\mathrm{H}_{2} \mathrm{O}$ ice permeated by spherical, gas-free pores. If these pores do not transport energy, they will reduce the overall conductivity of the medium. If $\mathrm{K}_{\mathrm{s}}$ is the bulk conductivity of solid ice, then the conductivity of the porous ice will be $\mathrm{K}=\phi \mathrm{K}_{\mathrm{s}}$, where $\phi<1$. The key problem is to determine the value of $\phi$. Smoluchowski (1981) suggested that this reduction would be proportional to the cross-sectional area of the pores. Since the volume of void is proportional to the porosity $\Psi$, its cross-sectional area should be proportional to $\Psi^{2 / 3}$. We might expect, therefore, that

$$
\phi \approx 1-\Psi^{2 / 3}
$$

In a later paper, Smoluchowski (1982) used a formula originally developed by Maxwell (1873). If we consider spherical grains of conductivity $\mathrm{K}_{\mathrm{p}}$ embedded in a matrix of conductivity $\mathrm{K}_{\mathrm{s}}$, and the grains occupy a small fraction of the total volume, $\Psi$, then the conductivity of the combined medium is reduced relative to that of the matrix by a factor

$$
\phi=\frac{(2-2 \Psi)+(1+2 \Psi) \frac{\mathrm{K}_{\mathrm{p}}}{\mathrm{K}_{\mathrm{s}}}}{(2+\Psi)+(1-\Psi) \frac{\mathrm{K}_{\mathrm{p}}}{\mathrm{K}_{\mathrm{s}}}}
$$

This formula is actually the first term in an expansion, and neglects effects of mutual "shadowing" by the grains. It is therefore exact only for the case of small $\Psi$. Higher-order terms were later added by Rayleigh (1892), which extended the applicability to larger values of $\Psi$. But precisely because the Maxwell formula neglects shadowing, it gives an upper limit to $\phi$. A lower limit can be obtained by inverting the problem: Let the matrix be composed of grain material and the grains be composed of ice. Then

$$
\phi_{1}=\frac{\mathrm{K}_{\mathrm{p}}}{\mathrm{K}_{\mathrm{s}}} \frac{2 \Psi \frac{\mathrm{K}_{\mathrm{p}}}{\mathrm{K}_{\mathrm{s}}}+(3-2 \Psi)}{(3-\Psi) \frac{\mathrm{K}_{\mathrm{p}}}{\mathrm{K}_{\mathrm{s}}}+\Psi}
$$

The spherical grains embedded in the ice matrix may be replaced by pores, in which case $\Psi$ is the porosity, and the pore conductivity due to the flux carried by radiation is

$$
\mathrm{K}_{\mathrm{p}}=4 \varepsilon \sigma \mathrm{r}_{\mathrm{p}} \mathrm{T}^{3}
$$

Squyres et al. (1985) used an expression due to Brailsford and Major (1964) for $\phi$ 


$$
\phi=\frac{K_{p}}{4 K_{s}}\left(A+\sqrt{A^{2}+\frac{8 K_{s}}{K_{p}}}\right)
$$

where

$$
\mathrm{A}=(2-3 \Psi) \frac{\mathrm{K}_{\mathrm{s}}}{\mathrm{K}_{\mathrm{p}}}+3 \Psi-1
$$

They also pointed out that if the matrix material is actually composed of ice grains, and the area of contact between adjoining grains is small, the resultant conductivity of the medium will be reduced even further by a so-called Hertz factor, the area of contact between material grains relative to the cross-sectional area. A different expression, known as Russel's formula, was used in other studies (see Espinasse et al., 1991)

$$
\phi=\frac{\Psi^{2 / 3} \frac{\mathrm{K}_{\mathrm{p}}}{\mathrm{K}_{\mathrm{s}}}+\left(1-\Psi^{2 / 3}\right)}{\Psi-\Psi^{2 / 3}+1-\Psi^{2 / 3}\left(\Psi^{1 / 3}-1\right) \frac{\mathrm{K}_{\mathrm{p}}}{\mathrm{K}_{\mathrm{s}}}}
$$

Steiner and Kömle (1991) proposed still another theoretical expression

$$
\begin{gathered}
\phi=(1-\sqrt{1-\Psi}) \Psi \frac{\mathrm{K}_{\mathrm{p}}}{\mathrm{K}_{\mathrm{s}}}+ \\
\sqrt{1-\Psi}\left[\varsigma+(1-\varsigma) \frac{\mathrm{B}+1}{\mathrm{~B}} \frac{\mathrm{K}_{\mathrm{p}}}{\mathrm{K}_{\mathrm{s}}+\mathrm{K}_{\mathrm{p}}}\right]
\end{gathered}
$$

where

$$
\mathrm{B}=1.25[(1-\Psi) / \Psi]^{10 / 9}
$$

is a deformation factor, and $\zeta$ is a flattening coefficient, which is essentially the Hertz factor. This factor depends on the details of the structure of the medium and cannot be determined $a$ priori.

Haruyama et al. (1993) and Sirono and Yamamoto (1997) used effective medium theory to derive

$$
\frac{\phi-1}{1+\left(\frac{1}{\Psi_{\mathrm{c}}}-1\right) \phi} \Psi+\frac{\phi-\frac{\mathrm{K}_{\mathrm{p}}}{\mathrm{K}_{\mathrm{s}}}}{\frac{\mathrm{K}_{\mathrm{p}}}{\mathrm{K}_{\mathrm{s}}}+\left(\frac{1}{\Psi_{\mathrm{c}}}-1\right) \phi}(1-\Psi)=0
$$

where $\Psi_{\mathrm{c}}$ is the percolation threshold of the medium (Stauffer and Aharony, 1994). None of these approaches, however, allows for a distribution of pore sizes, yet the pore-size distribution will certainly affect the resultant conductivity.

Recently, a new approach was adopted by Shoshany et al. (2002). Using a Monte Carlo procedure, they modeled a 3-D fractal medium made of ice and voids. A temperature gradient was assumed across this medium and the 3-D equations of heat transfer were solved to obtain the energy flux, which yields the effective conductivity. By running a series of models and fitting analytic functions to the results, this Monte Carlo approach allows one to find $\phi$ for a given porosity

$$
\begin{aligned}
\left(1-\Psi / \Psi_{\mathrm{c}}\right)^{\alpha(\Psi)} & \leq \phi \\
& \leq\left(1-\Psi_{\mathrm{m}} / \Psi_{\mathrm{c}}\right)^{\alpha\left(\Psi_{\mathrm{m}}\right) \ln (1-\Psi) / \ln \left(1-\Psi_{\mathrm{m}}\right)} \\
\alpha(\Psi) & =4.1 \Psi+0.22 \\
\Psi_{\mathrm{c}} & =0.7
\end{aligned}
$$

$\Psi_{\mathrm{m}}$ is the minimal possible porosity of the medium (essentially, its microporosity). If the pore size distribution is known as well, the value of $\phi$ within this range can be uniquely determined. A comparison of the different expressions is shown in Fig. 3.

The agreement goes only as far as the trend of decreasing conductivity with increasing porosity. The low conductivity of porous comet-analog material was also demonstrated experimentally (e.g., Benkhoff and Spohn, 1991; Kömle et al., 1996; Seiferlin et al., 1996). It should be mentioned that in addition to the pores that result from the grainy structure of the ice-dust matrix, there are almost certainly additional micropores in the ice. These may be inherent to the structure of ice formed by slow deposition of water vapor at low temperature (Kouchi et al., 1994), or may result from dissociation of clathrate-hydrates in the ice (Blake et al., 1991). They should have little direct influence on the conductivity of the medium (unless they are correlated in space and form cracks), and will not affect the density appreciably, but they will allow the gas to flow through the medium more freely.

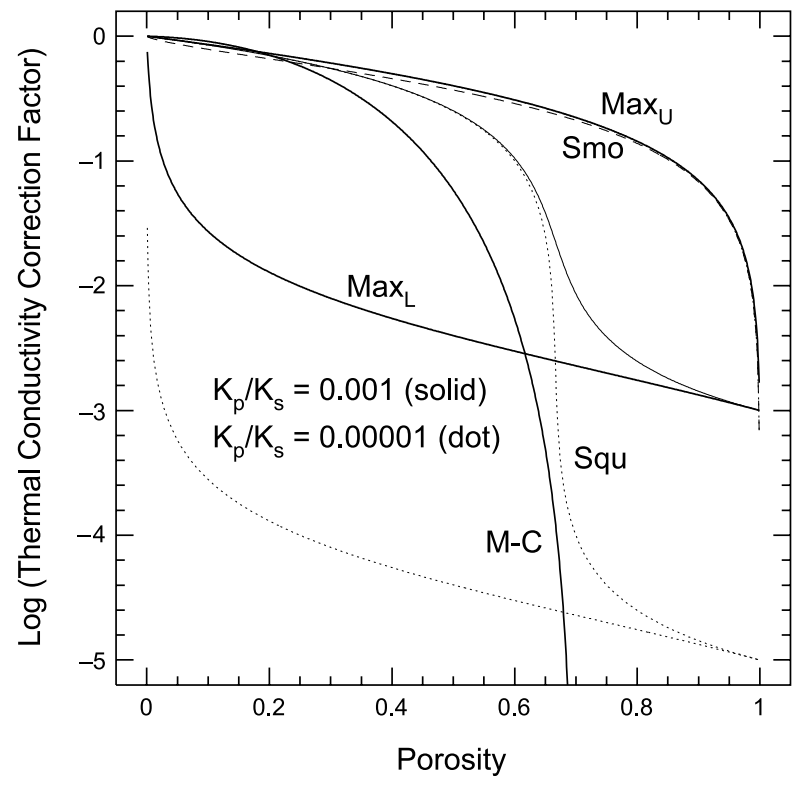

Fig. 3. Correction factor to the thermal conductivity resulting from porosity, as a function of porosity, for different models: Maxwell's upper $\left(\operatorname{Max}_{U}\right)$ and lower $\left(\operatorname{Max}_{\mathrm{L}}\right)$ limits, Smoluchowski's relation (Smo), Squyres et al.'s relation (Squ), and the Monte Carlo fractal model (M-C, see text). When applicable, two ratios of the pore to solid conductivity are considered, as shown on the figure. 


\subsection{Amorphous-Crystalline Transition in $\mathrm{H}_{2} \mathrm{O}$ Ice}

Ordinary ice has a crystalline structure, but when water vapor condenses at low temperatures, the molecules do not have sufficient energy to take up the proper sites in the crystal, and an amorphous material is produced. The thermal conductivity of amorphous ice (see Table 3 ) is much lower than that of crystalline ice, and its temperature dependence has an opposite trend, $\mathrm{K} \propto \mathrm{T}$ rather than $\mathrm{K} \propto \mathrm{T}^{-1}$. Experiments by Kouchi et al. (1992) indicate that the conductivity may be as much as four orders of magnitude lower. These authors suggest that their low value may be the result of the very slow deposition procedure used, which creates a network of connected micropores. This network, which is actually a system of cracks, will leave "islands" of disconnected amorphous ice that will substantially lower the conductivity. A similar behavior is observed in amorphous semiconductors. Again, we see that porosity effects may significantly alter the conductivity of a material.

Amorphous ice is unstable and tends to spontaneously convert to crystalline ice. Measurements by Schmitt et al. (1989) have shown that the rate of crystallization is given by

$$
\lambda(\mathrm{T})=1.05 \times 10^{13} \mathrm{e}^{-5370 / \mathrm{T}} \mathrm{s}^{-1}
$$

Crystallization has a number of consequences. First, the conductivity through the medium will change with time. This will be due mainly to the intrinsic difference in conductivity between the two phases. In addition, however, the two phases differ in density, as amorphous ice is denser than crystalline ice by $2-7 \%$. The precise difference depends on the rate of deposition [see Jenniskens and Blake (1994) and references therein], but in any case the phase change will subject the medium to stresses that may cause a change in porosity. This will further affect the thermal conductivity.

A second consequence comes from the fact that amorphous ice has the ability to trap volatiles. Extensive studies by Bar-Nun and co-workers [see Bar-Nun and Owen (1998) and references therein] have explored the dependence of the composition of the trapped gases, their relative abundances, and their rates of release on the temperature of deposition of the ice, and on the rate at which the medium is heated. In particular, they find that gas release accompanies the change in crystal structure. The amorphous-crystalline phase change should therefore lead to an increase in the activity of the nucleus.

Finally, the phase change releases latent heat, and this provides an internal heat source for the medium. The measured value is $\mathcal{H}_{\mathrm{ac}}=9 \times 10^{4} \mathrm{~J} \mathrm{~kg}^{-1}$ (Klinger, 1981). As the phase change is irreversible, this heat source is a sporadic one, and occurs only once in any given mass element of the comet.

\subsection{Radioactive Heat Production}

The most potent radioenergy source for comets is the short-lived radionuclide ${ }^{26} \mathrm{Al}$, which gained renewed interest owing to the detection of interstellar $1.809 \mathrm{MeV} \gamma$-rays from the decay of ${ }^{26} \mathrm{Al}$ (e.g., Mahoney et al., 1984). Furthermore, Srinivasan et al. (1999) detected for the first time ${ }^{26} \mathrm{Mg}$ in a differentiated meteorite, and therefore could confirm the role of ${ }^{26} \mathrm{Al}$ in the differentiation of meteoritic parent bodies. Thus it is already widely used in thermal modeling of asteroids [see Merk et al. (2002) and references therein]. Indeed, for small silicate bodies such as asteroids, accretion models predict growth times in the range between $10^{4} \mathrm{yr}$ and $1 \mathrm{~m} . \mathrm{y}$., depending on whether conditions allow for runaway accretion or not. Hence the comparably short lifetime of ${ }^{26} \mathrm{Al}$ and the growth time of asteroidal bodies are compatible. Comets, on the other hand, formed much farther from the Sun and hence took longer to accrete. But even in this case formation times may have been sufficiently short to allow for live ${ }^{26} \mathrm{Al}$. For example, Weidenschilling (1997) showed that it is possible to grow large icy bodies of a radius of about $40 \mathrm{~km}$ within $2.5 \times 10^{5} \mathrm{yr}$ in the region at $30 \mathrm{AU}$. All the observational evidence points toward an interstellar isotopic ratio of ${ }^{26} \mathrm{Al} / 27 \mathrm{Al} \approx 5 \times 10^{-5}$, implying an initial mass fraction $\mathrm{X}_{0}(26 \mathrm{Al}) \approx 7 \times 10^{-7}$ in dust (rock) and presumably an order of magnitude less in objects whose time of aggregation did not exceed a few million years.

The rate of heating by a radioactive isotope of relative abundance $X_{\text {rad }, 0}$ within the dust is given by

$$
\dot{\mathrm{Q}}_{\mathrm{rad}}=\rho_{\mathrm{d}} \mathrm{X}_{\mathrm{rad}, 0} \mathcal{H}_{\mathrm{rad}} \tau_{\mathrm{rad}}^{-1} \mathrm{e}^{-\mathrm{t} / \tau_{\mathrm{rad}}}
$$

where $\mathcal{H}_{\text {rad }}$ is the energy released per unit mass upon decay and $\tau_{\text {rad }}$ is the decay time constant. The total contribution is obtained by summing over the different species. The major long-lived sources of radioactive heating $-{ }^{40} \mathrm{~K},{ }^{232} \mathrm{Th}$, ${ }^{235} \mathrm{U}$, and ${ }^{238} \mathrm{U}$ - together provide some $3 \times 10^{-11} \mathrm{~J} \mathrm{~kg}^{-1} \mathrm{~s}^{-1}$. The short-lived ${ }^{26} \mathrm{Al}$, assuming an initial mass fraction $\mathrm{X}_{0} \sim$ $5 \times 10^{-8}$, could have provided as much as $2 \times 10^{-8} \mathrm{~J} \mathrm{~kg}^{-1} \mathrm{~s}^{-1}$.

\subsection{Sublimation and the Surface/Volume Ratio}

The porous structure of the comet nucleus allows for an internal process that is otherwise confined to the cometary surface: sublimation of ice from the pore walls or condensation onto them. The rate of sublimation - mass per unit volume of cometary material per unit time - is given by

$$
\mathrm{q}_{\alpha}=\mathrm{S}\left(\Psi, \mathrm{r}_{\mathrm{p}}\right)\left[\left(\mathcal{P}_{\alpha}(\mathrm{T})-\mathrm{P}_{\alpha}\right) \sqrt{\frac{\mu_{\alpha}}{2 \pi \mathcal{R}_{\mathrm{g}} \mathrm{T}}}\right]
$$

where the term in square brackets represents the sublimation rate per unit surface area. Thus the property of the porous structure that affects sublimation is the surface to volume ratio $\mathrm{S}$, defined as the total interstitial surface area of the pores $\mathrm{A}_{\mathrm{p}}$ per given bulk volume $\mathrm{V}$

$$
\mathrm{S}=\mathrm{A}_{\mathrm{p}} / \mathrm{V}
$$

Its evaluation requires some model of the porous configuration. As a simple example, consider the specific surface of a porous material made of identical spheres of radius $r_{s}$ 
in a cubical packing. In this case $A_{s}=4 \pi r_{s}^{2} N$, where $N$ is the number of spheres in the given volume V. Obviously, V = $\left(2 r_{s}\right)^{3} \mathrm{~N}$, which yields $S=\pi / 2 r_{s}$. If the solid spheres are replaced by spherical pores embedded in a solid matrix, the result is the same. Clearly, fine materials have a much greater specific surface than coarse materials. Consider now a more realistic case of a granular medium of spherical grains of $n$ different sizes, so that the number of grains of radius $r_{i}(1 \leq$ $\mathrm{i} \leq \mathrm{n}$ ) is $\mathrm{N}_{\mathrm{i}}$. The total area and volume of these spheres are

$$
\begin{gathered}
A_{s}=\sum_{i=1}^{n} 4 \pi r_{i}^{2} N_{i}=A_{p} \\
V_{s}=\sum_{i=1}^{n} \frac{4}{3} \pi r_{i}^{3} N_{i}
\end{gathered}
$$

respectively. By definition, $\mathrm{V}=\mathrm{V}_{\mathrm{s}} /(1-\Psi)$, whence

$$
\mathrm{S}=3(1-\Psi) \sum_{\mathrm{i}=1}^{\mathrm{n}} f_{\mathrm{i}} / \mathrm{r}_{\mathrm{i}}=3(1-\Psi) / \mathrm{r}_{\mathrm{p}}
$$

where $r_{p}$ is the harmonic mean radius weighted by $f_{\mathrm{i}}$, the volume fraction occupied by spheres of radius $r_{i}$. As before, pores and grains may be interchanged.

Another case, often used in comet nucleus modeling, is that of a bundle of cylindrical tortuous capillary tubes that do not cross each other (Mekler et al., 1990). The tortuosity is defined as the ratio of the capillary length to the sampled thickness. For a given length $\mathrm{L}$ and unit cross-sectional area we have

$$
\begin{gathered}
A_{p}=\sum_{i=1}^{n} 2 \pi r_{i} N_{i} \xi L \\
V=1 \cdot L
\end{gathered}
$$

where $r_{i}$ is the capillary radius $(1 \leq \mathrm{i} \leq \mathrm{n}), \mathrm{N}_{\mathrm{i}}$ is the number of capillaries of radius $r_{i}$ crossing a unit area, and $\xi$ is the typical capillary tortuosity. Thus

$$
S=\sum_{i=1}^{n} 2 \pi r_{i} N_{i} \xi
$$

On the other hand,

$$
\Psi=\sum_{\mathrm{i}=1}^{\mathrm{n}} \pi \mathrm{r}_{\mathrm{i}}^{2} \mathrm{~N}_{\mathrm{i}} \xi
$$

which leads to

$$
\mathrm{S}=2 \Psi \sum_{\mathrm{i}=1}^{\mathrm{n}} f_{\mathrm{i}} / \mathrm{r}_{\mathrm{i}}=2 \Psi / \mathrm{r}_{\mathrm{p}}
$$

where $r_{p}$ is the harmonic mean radius weighted by $f_{i}$, the volume fraction occupied by capillaries of radius $r_{i}$.
We note that the two models behave differently with changing porosity: As $\Psi$ decreases, the surface to volume ratio of capillaries tends to zero, while that of spheres increases to a maximum. Toward high porosities, on the other hand, the surface to volume ratio of spheres tends to zero. It is, however, difficult to visualize either a low-porosity medium made up of a bundle of individual capillaries, or a high-porosity one made up of widely separated spheres. Therefore, in numerical modeling that allows for a changing porosity - due, for example, to vigorous sublimation or condensation - it would be advisable to change from one model to the other as the porosity changes. The models yield equal values of $\mathrm{S}$ for $\Psi=0.6$. Among other relations that have been suggested in the literature, we find (Kaponen et al., 1997)

$$
S \propto-\Psi \ln \Psi
$$

Beside the major ice component $\mathrm{H}_{2} \mathrm{O}$, comet models usually include several other components of higher volatility $\left(\mathrm{CO}, \mathrm{CH}_{4}, \mathrm{CO}_{2}, \mathrm{CH}_{3} \mathrm{OH}, \mathrm{HCN}, \mathrm{NH}_{3}, \mathrm{H}_{2} \mathrm{~S}, \mathrm{C}_{2} \mathrm{H}_{2}\right.$, $\mathrm{C}_{2} \mathrm{H}_{6}, \mathrm{C}_{3} \mathrm{H}_{4}$ ). For each species, an empirical formula for the saturated vapor pressure is used, of the form

$$
P=\mathrm{Ae}^{-\mathrm{B} / \mathrm{T}}
$$

The coefficients for A and B for different ices are given in Table 4. The coefficients for water and carbon monoxide may be found in Fanale and Salvail (1984). The other parameters are extrapolated from fits to data found in the Handbook of Chemistry and Physics (Lide, 2003).

The latent heat of sublimation $\mathcal{H}$ is calculated from the Clausius-Clapeyron equation

$$
\frac{1}{\mathrm{P}} \frac{\partial \mathrm{P}}{\partial \mathrm{T}}=\frac{\mu \mathcal{H}}{\mathrm{T}^{2} \mathcal{R}_{\mathrm{g}}}
$$

Using the empirical equation (44), we obtain $\mathrm{B}=\mu \mathcal{H} / \mathcal{R}_{\mathrm{g}}$, implying constant values for $\mathcal{H}$.

TABLE 4. List of coefficients for the pressure equation.

\begin{tabular}{lccc}
\hline Ice Component & Symbol & $\begin{array}{c}\text { A Value } \\
\left(10^{10} \mathrm{Nm}^{-2}\right)\end{array}$ & $\begin{array}{c}\text { B Value } \\
\text { (Kelvin) }\end{array}$ \\
\hline Water & $\mathrm{H}_{2} \mathrm{O}$ & 356. & 6141.667 \\
Carbon monoxide & $\mathrm{CO}$ & 0.12631 & 764.16 \\
Carbon dioxide & $\mathrm{CO}_{2}$ & 107.9 & 3148 \\
Methane & $\mathrm{CH}_{4}$ & 0.597 & 1190.2 \\
Propyne & $\mathrm{C}_{3} \mathrm{H}_{4}$ & 3.417 & 3000 \\
Propadine & $\mathrm{C}_{3} \mathrm{H}_{4}$ & 2.382 & 2758 \\
Ethane & $\mathrm{C}_{2} \mathrm{H}_{6}$ & 0.459 & 1938 \\
Methanol & $\mathrm{CH}_{3} \mathrm{OH}$ & 8.883 & 4632 \\
Hydrogen cyanide & $\mathrm{HCN}$ & 3.8665 & 4024.66 \\
Hydrogen sulphide & $\mathrm{H}_{2} \mathrm{~S}$ & 1.2631 & 2648.42 \\
Ammonia & $\mathrm{NH}_{3}$ & 61.412 & 3603.6 \\
Acetylene & $\mathrm{C}_{2} \mathrm{H}_{2}$ & 9.831 & 2613.6 \\
\hline
\end{tabular}




\subsection{Gas Flow and Permeability}

The gas released in the interior of the nucleus, either by sublimation from the pore walls or as a result of crystallization of amorphous ice, will diffuse through the pores. Flow through a porous medium will depend on the properties of the medium and the properties of the flowing material itself. A simple formulation for fluid flow through a porous medium was derived experimentally by Darcy as early as 1856 and has become known as Darcy's law

$$
\mathbf{J} \propto \frac{1}{\mathrm{v}} \nabla \mathrm{P}
$$

where $v$ is the kinematic viscosity of the fluid; in the case of an ideal gas

$$
v \approx \frac{1}{3} \ell v_{\text {th }}
$$

and

$$
\mathrm{v}_{\mathrm{th}}=\sqrt{\frac{8 \mathrm{kT}}{\pi \mathrm{m}}}
$$

The simple law (equation (46)) is, however, only approximately correct.

The flow regime for a gas in a porous medium is determined by the Knudsen number defined as the ratio of the mean free path of a gas molecule to the pore diameter

$$
\mathrm{Kn}=\frac{\ell}{2 \mathrm{r}_{\mathrm{p}}}
$$

If $\mathrm{n}$ is the gas number density and $\sigma_{\alpha}$ the kinetic cross section of a gas molecule, then

$$
\ell=\frac{1}{\sigma_{\alpha} \mathrm{n}}=\frac{\mathrm{kT}}{\sigma_{\alpha} \mathrm{P}}
$$

(Note that the cross section is usually defined as $\pi \mathrm{d}^{2}$, where $\mathrm{d}$ is the molecular diameter; it may also include a factor $\sqrt{2}$ if velocity effects are taken into account.) The highest ice temperature attained in comet nuclei is of the order of $200 \mathrm{~K}$; substituting in equation $(50) \sigma_{\mathrm{H}_{2} \mathrm{O}} \approx 2.5 \times 10^{-15} \mathrm{~cm}^{2}$ and $\mathrm{P} \approx \mathcal{P}_{\mathrm{H}_{2} \mathrm{O}}(200 \mathrm{~K})$, we obtain $\ell \approx 4 \mathrm{~cm}$. Hence so long as the average pore size is less than $1 \mathrm{~mm}$, we have $\mathrm{Kn} \gg 1$, meaning that the flow of gas through the pores is a free molecular, or Knudsen flow, where collisions of the gas particles with the pore walls are much more frequent than collisions between particles. In this regime, the amount of mass passing through a cylindrical tube per unit time is given by

$$
\mathbf{j}=-\frac{8 \mathrm{r}_{\mathrm{p}}^{3}}{3 \xi} \sqrt{\frac{\pi \mathrm{m}}{2 \mathrm{k}}} \nabla(\mathrm{P} / \sqrt{\mathrm{T}})
$$

(see Mekler et al., 1990). Adopting again the model of a bundle of capillaries, we obtain for the mass flux

$$
\mathbf{J}_{\mathrm{Kn}}=-\frac{8}{3} \Phi\left(\frac{\mathrm{m}}{2 \pi \mathrm{k}}\right)^{1 / 2} \nabla(\mathrm{P} / \sqrt{\mathrm{T}})
$$

where $\Phi$, defined as the permeability of the medium, is obtained by using equation (41) and the same weighting function as in the calculation of $\mathrm{S}$ for the same model (equation (42))

$$
\Phi=\Psi r_{p} / \xi^{2}
$$

Another common relation between $\Phi$ and $\Psi$, known as the Kozeny law, is of the general form

$$
\Phi \propto \Psi^{3} /\left(\xi^{2} S^{2}\right)
$$

For a relatively low porosity, near the percolation limit $\Psi_{c}$, below which there is no continuous flow through the medium, the relation is of the form

$$
\begin{gathered}
\Phi \propto\left(\Psi-\Psi_{\mathrm{c}}\right)^{\mu} \\
\mu=2.8
\end{gathered}
$$

However, when the pore size is increased, the condition $\mathrm{Kn}>1$ may no longer apply. The flow becomes a continuum (Poiseuille), or viscous flow, dominated by collisions between particles

$$
\mathbf{J}_{\mathrm{Po}}=-\frac{3}{16} \frac{\Psi_{\mathrm{p}}^{2} \sigma_{\alpha}}{\xi^{2}}\left(\frac{\mathrm{m} \pi}{2 \mathrm{k}^{3}}\right)^{1 / 2} \frac{\mathrm{P}}{\mathrm{T}^{3 / 2}} \nabla \mathrm{P}
$$

For the intermediate regime, $\mathrm{Kn} \sim 1$, semiempirical interpolation formulae are commonly used, of the general form $\mathbf{J}=\mathrm{a}_{1} \mathbf{J}_{\mathrm{Kn}}+\mathrm{a}_{2} \mathbf{J}_{\mathrm{Po}}$, known as the Adzumi equation, with fixed (empirically determined) coefficients $a_{1}$ and $a_{2}$. Each one of the flow equations is suitable for a set of given conditions. But when $\mathrm{Kn}$ is neither uniform nor constant, as in the case of an evolving comet, the above formulae do not ensure a smooth transition between the two flow regimes as the Knudsen number changes from $\mathrm{Kn} \gg 1$ to $\mathrm{Kn} \ll 1$. A more suitable interpolation may be obtained by noting that if the temperature is uniform, equation (52) reduces to an expression similar in form to equation (56). This suggests an interpolation similar to the Adzumi equation

$$
\mathbf{J}=\left(1+\frac{9 \pi}{256} \frac{1}{\mathrm{Kn}}\right) \mathbf{J}_{\mathrm{Kn}}
$$

which varies continuously from $\mathbf{J}_{\mathrm{Kn}}$ for $\mathrm{Kn} \gg 1$ to $\mathbf{J}_{\mathrm{Po}}$ for $\mathrm{Kn} \ll 1$.

When two gases (e.g., $\mathrm{H}_{2} \mathrm{O}$ vapor and $\mathrm{CO}$ ) are flowing through the same medium, they are treated independently, namely each flux is computed according to equation (57). 
Since the molecular flow is driven by the partial pressure, whereas the viscous flow is driven by the total pressure, this is strictly correct in the Knudsen regime, and also in the case of immiscible flows (see Bouziani and Fanale, 1998). Fortunately, the flux of initially trapped gas is overwhelmingly dominant in the interior of the comet, while the $\mathrm{H}_{2} \mathrm{O}$ flux becomes dominant in a very thin outer layer of the nucleus (a few centimeters), where most of the sublimation occurs. The interaction between gases is therefore restricted to this layer. At high flow rates turbulence may arise, and equation (57) may no longer hold. This is indicated by the Reynolds number exceeding a critical value of order 1000 . When the Reynolds number is routinely evaluated during evolutionary calculations, it is always found to remain smaller than 100 , and therefore equation (57) may be safely applied.

\subsection{Drag on Dust Grains}

As the ice sublimates from the porous nucleus matrix, dust grains are released into the gas stream. Transport of dust must therefore be considered in addition to gas flow, a problem that is only now beginning to be studied.

We have seen that the flow of gas through porous comet nuclei is typically a free molecular (Knudsen) flow. The drag force on a dust grain of radius $r_{d}$ in the Knudsen regime is

$$
\mathrm{F}_{\mathrm{drag}} \approx 2 \pi \mathrm{r}_{\mathrm{d}}^{2} \rho \mathrm{v}_{\mathrm{th}}\left(\mathrm{v}_{\mathrm{g}}-\mathrm{v}_{\mathrm{d}}\right)
$$

up to a numerical factor of order unity (Öpik, 1958), where $\mathrm{v}_{\mathrm{g}}$ is the gas velocity and $\mathrm{v}_{\mathrm{d}}$ is the dust grain velocity. Combining equations (58) and (48) and dividing by the mass of the (spherical) dust grain, we obtain the grain's acceleration

$$
\frac{\mathrm{dv}_{\mathrm{d}}}{\mathrm{dt}}=\frac{1}{\tau\left(\mathrm{r}_{\mathrm{d}}\right)}\left(\mathrm{v}_{\mathrm{g}}-\mathrm{v}_{\mathrm{d}}\right)
$$

where $\varrho_{d}$ is the density of the grain and the characteristic time $\tau$, a function of the dust grain radius for given flow conditions, is

$$
\tau \approx \frac{\varrho_{\mathrm{d}}}{\rho} \sqrt{\frac{\mathrm{m}}{\mathrm{kT}}} \mathrm{r}_{\mathrm{d}}
$$

The dust grain velocity (assuming a constant gas velocity) is thus

$$
v_{d}(t)=v_{g}\left(1-e^{-t / \tau}\right)
$$

For conditions that are typical of cometary interiors (a few meters to a few tens of meters below the surface), where crystallization of amorphous ice takes place and trapped gas is released, or where volatile species (such as $\mathrm{CO}$ ) sublimate, we find $\tau \approx 0.5\left(\mathrm{r}_{\mathrm{d}} / 1 \mu \mathrm{m}\right) \mathrm{s}$, so that even $10-\mu \mathrm{m}$ particles can reach $90 \%$ of the gas velocity in about $10 \mathrm{~s}$. For gas velocities typical of such conditions, the particle will have traveled during that time interval a distance of much less than $1 \mathrm{~m}$. This length scale is considerably smaller than the typical length scale over which conditions change in the interior of the nucleus. Near the surface, where the main driving force is provided by water vapor sublimating from the pore walls, conditions are even more favorable.

So far we have neglected the effect of gravity. A gravitational acceleration $g$ would change the solution in equation (61) for the velocity to

$$
v_{d}(t)=\left(v_{g}-g \tau\right)\left(1-e^{-t / \tau}\right)
$$

Hence the effect is negligible so long as $g \tau \ll v$. For a constant nucleus density $\rho$, and at depths that are much smaller than $R$, we have $g=(4 \pi / 3) G \rho R$. For parameters characteristic of cometary interiors (resulting in the above estimate of $\tau$ ) this condition is amply satisfied. It will break down for very large dust grains (see discussion of critical radius in the next section), but the flow of such grains would in any case be prevented by the small pore size.

In conclusion, to a good approximation, the dust grain can be taken to move at the same speed as the gas. The difference is that while the gas can move fairly freely through the nucleus by diffusing through pores (or, if need be, through micropores), a dust grain can only move through those pores that are large enough to accommodate it. Several models have been suggested to treat this problem. Podolak and Prialnik (1996) proposed that the dust motion be treated as a random walk. Shoshani et al. (1997) treat the porous medium as a sequence of filters, each with a size distribution of holes. The pores are viewed as cylinders extending from these holes. The distance between filters is the average distance one would travel in a cylindrical pore before the radius of the pore changed significantly. Assuming a given dust grain speed and given size distributions for grains and for pores, they follow the change in dust size distribution as the dust migrates through the nucleus. They also show how the trapping of dust grains affects the porosity and permeability of the medium.

More recently, Shoshany et al. (1999) used Monte Carlo calculations to explore the behavior of dust migration in a medium with randomly distributed pores. They found that the effective speed of dust particles is lower than that found by the random walk model for all porosities, although the difference decreases for $\Psi \rightarrow 0$ or $\Psi \rightarrow 1$ as expected. They also found that only the smallest dust grains (of order of the pore size) traversed the medium for any distance. Larger grains could not find sufficiently many large pores to travel freely, and they got trapped after moving only a short distance. Large grains that are observed in a comet coma were most likely lifted off directly from the surface. Smaller grains may have a component originating deeper in the nucleus. The exponent obtained from fitting a power law to the observed grain size distribution in the coma may therefore not accurately reflect the grain size distribution within the nucleus itself.

Recent studies (Skorov and Rickman, 1995, 1998, 1999) have begun to focus on the details of the interaction of the gas flow with the individual dust grains. These Monte Carlo computations follow the flow of gas molecules in the Knudsen regime as they leave the surface of the nucleus and 
interact with the dust grains above it. This work studies the gas kinetic flow as a function of capillary length, inclination angle, and temperature gradient along the pores at the surface of the nucleus. It also follows the velocity distribution of the gas molecules, and how it is affected by interaction with the dust grains. Like all Monte Carlo computations, this program is advancing slowly, but is beginning to produce important insight into the gas-dust interaction.

How do these studies contribute to the numerical simulation of a comet nucleus evolution? As the effective rate of flow of dust particles clearly depends on the particle size, we may assume in numerical calculations the dust grain radii to be distributed over a discrete range $r_{d, 1}, r_{d, 2}, \ldots r_{d, N}$, according to some distribution function $\psi\left(\mathrm{r}_{\mathrm{d}}\right)$ (such as a power law). The size of a dust particle may be assumed to remain unchanged, thus ignoring possible breakup or coalescence of dust grains. Hence particles in each size category may be treated as independent species. The local flux of dust particles of radius $r_{d, n}$ is therefore given by

$$
\mathrm{J}_{\mathrm{d}, \mathrm{n}}=\beta_{\mathrm{n}} \rho_{\mathrm{d}, \mathrm{n}} \mathrm{v}_{\mathrm{g}}
$$

It is the coefficient $\beta_{\mathrm{n}}$ that must be determined by the dust flow model (cf. Horanyi et al., 1984). For example, Podolak and Prialnik (1996) adopt

$$
\beta_{\mathrm{n}} \propto \log \left[1-\psi\left(\mathrm{r}_{\mathrm{d}, \mathrm{n}}\right)\right] / \log \left[\left(1-\psi\left(\mathrm{r}_{\mathrm{d}, \mathrm{n}}\right)\right) \psi\left(\mathrm{r}_{\mathrm{d}, \mathrm{n}}\right)\right]
$$

The mass conservation equation for these particles is

$$
\frac{\partial \rho_{\mathrm{d}, \mathrm{n}}}{\partial \mathrm{t}}+\nabla \cdot \mathbf{J}_{\mathrm{d}, \mathrm{n}}=0
$$

We note that there is no source term in equation (63); the implicit assumption in this simple approximation is that any dust grain that can be dragged (allowance being made for the critical radius and the local average pore radius) is dragged with the gas.

The results expected from any dust flow model are the grain size distribution of the ejected dust and the changing pore structure of the medium through which the dust flows. For example, the large dust grains left behind on the nucleus surface form a dust mantle, which, in turn, affects the rate of heat and gas flow at the surface.

\subsection{Dust Mantle Formation}

The eventual formation of a dust mantle on the surface of a comet nucleus may be modeled in different ways, the essential parameter being the critical dust grain radius, which represents the radius of the largest particle that can leave the comet, as determined by the balance of forces acting on a dust grain. The drag force exerted by the gas flux at the surface is

$$
\mathrm{F}_{\mathrm{drag}}=\frac{1}{2} \mathrm{C}_{\mathrm{D}} \pi \mathrm{r}_{\mathrm{d}}^{2} \sum_{\alpha} \mathrm{v}_{\alpha} \mathrm{J}_{\alpha} \approx \pi \mathrm{r}_{\mathrm{d}}^{2} \sum_{\alpha} \mathrm{v}_{\alpha} \mathrm{J}_{\alpha}
$$

summing over the different species that contribute to the overall gas flux and adopting, in the lowest approximation, a drag coefficient $\mathrm{C}_{\mathrm{D}} \approx 2$. When sublimation at the surface is the dominant component, then equation (64) reduces to $\pi \mathrm{r}_{\mathrm{d}}^{2} \mathcal{P}(\mathrm{T})$. The effective gravitational force, diminished by the centrifugal force, is

$$
\mathrm{F}_{\text {grav }}=\frac{4 \pi}{3} \mathrm{r}_{\mathrm{d}}^{3} \varrho_{\mathrm{d}} \mathrm{g}\left(1-\frac{3 \pi \cos ^{2} \theta}{\mathrm{G \rho P}_{\text {spin }}^{2}}\right)
$$

with $g=4 \pi \mathrm{G} \rho \mathrm{R} / 3$, but the correction term is small so long as $\mathrm{P}_{\text {spin }}<3 \mathrm{~h}$. Thus roughly

$$
\mathrm{r}_{\mathrm{d}}^{*} \approx \frac{P(\mathrm{~T})}{\mathrm{G} \rho \varrho_{\mathrm{d}} \mathrm{R}}
$$

The problem of dust mantle formation was first studied by Brin and Mendis (1979), who related the mantle thickness $\mathrm{D}$ at a particular point in the orbit to its thickness at an earlier point by

$$
\begin{gathered}
D\left[d_{H}(t)\right]=D\left[d_{H}(t-\delta t)\right](1-\Theta)+ \\
(1-\Omega) X_{d} \frac{Q}{\varrho_{d}} \delta t
\end{gathered}
$$

where $\Omega$ is the fraction of dust released from ice and carried away by the sublimating gas, and $\Theta$ is the additional part of the mantle removed by the increased gas flux. For a grain size distribution $\psi\left(\mathrm{r}_{\mathrm{d}}\right)$ within a range $\left[\mathrm{r}_{\mathrm{d}}^{\min }, \mathrm{r}_{\mathrm{d}}^{\max }\right]$ and a critical grain size $r_{d}^{*}(t)$, which changes along the orbit, the functions $\Omega$ and $\Theta$ can be calculated and thus the development and evolution of this dust mantle can be followed. The rate of growth of the mantle is determined by the parameter $(1-\eta)$, where

$$
\eta=\frac{\int_{r_{d}^{\min }}^{r_{d}^{*}} \psi\left(r_{d}\right) r_{d}^{3} d r_{d}}{\int_{r_{d}^{\min }}^{r_{d}^{\max }} \psi\left(r_{d}\right) r_{d}^{3} d r_{d}}
$$

This simple approach deals, however, only with the mass of the mantle, regardless of its structure.

One approach to modeling the structure of the dust mantle is to assume that the ice sublimates freely at the nucleus surface, carrying with it the smaller (than critical size) dust grains, while the larger grains are left behind. At the beginning, these large dust grains are isolated from each other, but as more and more grains accumulate, the surface becomes evenly covered and starts interfering with the escape of smaller and smaller grains. The porosity of the dust mantle decreases and eventually drops below that of the nucleus. This idea of trapping and compaction, introduced by Shul'man (1972), was adopted by Rickman et al. (1990) in modeling the dust mantle. They showed that if the grain size distribution follows a power law with an index of about -3.5 , the smallest grains left behind contribute the most to forming the dust mantle. The gas flow through such a mantle can be modeled by considering gas diffusion through this porous medium. If the gas pressure is high enough, the 
dust mantle can be blown off, and the process will start anew. The process depends both on latitude and on the inclination of the rotation axis. A dust mantle will inhibit gas sublimation when most of the surface, close to $100 \%$, is covered by grains (e.g., Prialnik and Bar-Nun, 1988), a result that was confirmed by the KOSI experiments (Grün et al., 1993).

Eventually, the pore size of the dust mantle may become too small to allow particles to escape and a large amount of small grains will become permanently trapped. This may lead to a very stable and efficient dust crust with a high cohesive strength, which may surpass the vapor pressure building up in the porous material underneath the mantle (Kührt and Keller, 1994). As a consequence, the gas is driven toward the interior and refreezes, forming an ice layer of increased density (Prialnik and Mekler, 1991). This effect was actually observed in the KOSI comet simulation experiments (Spohn et al., 1989).

\subsection{Tensile Strength and Fracture of the Nucleus}

The material strength of comet nuclei is very low. Although the range of values resulting from different estimates is wide, all values indicate a weak material. The strength deduced from tidal breakup of Sun-grazing comets is $10^{2}$ $10^{4} \mathrm{~Pa}$ (Sekanina, 1984; Klinger et al., 1989). Laboratory experiments lend further support to the low strength estimates derived from observations: The typical strengths of the ice crusts measured in the KOSI experiments were in the 105-Pa range (Kochan et al., 1989).

A simple model for the strength of a medium composed of spherical grains was developed by Greenberg et al. (1995). Taking the nominal dipole-dipole interaction to be $\sim 10^{-2} \mathrm{eV}$, they obtain for the tensile strength

$$
\mathcal{T}=6.1 \times 10^{2}(1-\Psi) \beta\left(\frac{\mathrm{r}_{\mathrm{d}}}{0.1 \mu \mathrm{m}}\right)^{-2} \mathrm{~Pa}
$$

where $1 \leq \beta \leq 12$ is the number of contact points per particle. The strength and Young's modulus for a medium composed of ice grains linked into chains by intermolecular forces is computed by Sirono and Greenberg (2000). They show that these forces are strong enough to hold an assemblage of grains together even when its self-gravity will not. They derive $3 \times 10^{2} \mathrm{~Pa}$ for the tensile and $6 \times 10^{3} \mathrm{~Pa}$ for the compressive strength, when applied to the tidally split Comet Shoemaker-Levy 9.

Rotational stability against breakup of fast-rotating comets provide an independent means of estimating the strength of cometary material. Computations of this kind have been presented by Davidsson for solid spheres (Davidsson, 1999) and for biaxial ellipsoids (Davidsson, 2001). Cook (1989) considers a medium composed of bulk material permeated by cracks. The energy of such a medium will be a sum of the elastic strain energy in the bulk material and the surface energy along the crack. The crack will spread if the total energy of the material is decreased thereby. Cook applies this picture to a fractal material composed of successive generations of spherical aggregates. This picture may be useful for modeling the strength of comet nuclei.

In a weak porous medium, thermal stresses (Kührt, 1984) and internal pressure exerted by gas that accumulates in the pores may break the fragile solid matrix. For example, a model of a typical comet given in Prialnik et al. (1993) yields internal water vapor pressures exceeding $2 \times$ $10^{5} \mathrm{~Pa}$. This is comparable to the above strength estimates for cometary ice. If the stress, $\sigma_{\mathrm{m}}$, on a material exceeds its tensile strength $\mathcal{T}$, then that material will undergo tensile fracture. If the stress is negative (compression) and exceeds the compressive strength $C$, the material will undergo shear fracture. In a spherical shell of radius $r$, the tangential stress is given by

$$
\frac{\sigma_{\mathrm{m}}}{\mathrm{r}}=-\frac{1}{2} \frac{\mathrm{dP}}{\mathrm{dr}}
$$

(Morley, 1954), so that fracture should occur when

$$
-\frac{\mathrm{dP}}{\mathrm{dr}}>\frac{2 \mathcal{T}}{\mathrm{r}}
$$

In general $C \gg \mathcal{T}$, so that only tensile fracture needs to be considered. Prialnik et al. (1993) present a simple algorithm for dealing with this effect. They assume that the porosity of the medium remains unchanged, but the average pore radius increases as a result of fracture. When the local pressure gradient is high enough so that condition (70) is satisfied (for example, due to crystallization and release of trapped gas), the local average radius of the pores is increased by a factor proportional to $(\mathrm{r} / 2 \mathcal{T}) \mathrm{dP} / \mathrm{dr}$. Then a relaxation time is allowed so that the gas can flow through the enlarged pores, after which condition (70) is tested again. The energy of deformation of the matrix is small compared with the energy released by the amorphous-crystalline transition, and may be neglected. This procedure can also allow for the effect of strain hardening, whereby the strength of a material is increased due to deformation. In this case one can allow the tensile strength of the material to increase along with the increasing pore size.

In summary, as a result of fracture, the size distribution of pores will vary throughout the nucleus. In addition, sintering (Kossacki et al., 1999) or pore blocking by small dust grains may alter pore sizes as well as consolidate the material. Massive recondensation of volatiles on pore walls has a similar effect. A subject for future work is to incorporate additional processes such as grain growth by sintering and the elimination of pores by densification. An excellent review of the relevant processes is given by Eluszkiewicz et al. (1998). We are again faced with a complex internal process for which we must account based on very little information. Moreover, the pore size distribution measured at the surface of a comet nucleus need not represent the distribution in the deeper layers. However, extensive studies of crater 
formation in ice targets (Arakawa et al., 2000) have shown that the crater depth scales as the square root of the impact energy. More importantly, the crater pattern depends not only on the energy of the impactor, but also on the details of the layering of the ice. Thus future measurements of craters on comet nuclei may yield clues to their underlying structure.

\subsection{Characteristic Timescales}

Each process mentioned in this section has its own characteristic timescale and the competition between these timescales will determine to a large extent the evolutionary pattern of the comet nucleus that will be discussed in the next section:

1. The thermal timescale, obtained from the energy conservation equation (10), which in its simplest form (without sources and advection) is a heat-diffusion equation. Distinction must be made between the thermal timescale of amorphous ice $\tau_{\mathrm{a}-\mathrm{ice}}$, crystalline ice $\tau_{\mathrm{c}-\mathrm{ice}}$, and dust $\tau_{\text {dust }}$. For a layer of thickness $\Delta \mathrm{r}$ and average temperature $\mathrm{T}$ we have

$$
\tau_{\mathrm{a}-\text { ice }}=(\Delta \mathrm{r})^{2} \rho_{\mathrm{a}} \mathrm{c}(\mathrm{T}) / \mathrm{K}_{\mathrm{a}}
$$

and similar expressions for $\tau_{\mathrm{c}-\mathrm{ice}}$ and $\tau_{\mathrm{dust}}$. As a rule, $\tau_{\mathrm{c}-\text { ice }}<$ $\tau_{\mathrm{a}-\mathrm{ice}}<\tau_{\text {dust }}$; as we have seen in section 3.1 , porosity increases all these timescales considerably.

2. The timescale of gas diffusion $\tau_{\text {gas }}$, which is also the timescale of pressure release, obtained from the mass conservation equation, which (without sources) can be regarded as a diffusion-type equation for the release of gas pressure:

$$
\tau_{\mathrm{gas}}=\frac{3}{4} \frac{(\Delta \mathrm{r})^{2}}{\Psi \mathrm{r}_{\mathrm{p}}}\left(\frac{2 \pi \mathrm{m}}{\mathrm{kT}}\right)^{1 / 2}
$$

3. The timescale of crystallization $\tau_{\mathrm{ac}}$, which is also the timescale of gas-release and pressure buildup:

$$
\tau_{\mathrm{ac}}=\lambda(\mathrm{t})^{-1}=9.54 \times 10^{-14} \mathrm{e}^{5370 / \mathrm{T}} \mathrm{s}
$$

4. The timescales of sublimation of the different volatiles, $\tau_{\text {subl- } \mathrm{H}_{2} \mathrm{O}}$ for water, $\tau_{\text {subl-CO }}$ for $\mathrm{CO}$ and $\tau_{\text {subl-CO}}$ for $\mathrm{CO}_{2}$, and so forth:

$$
\tau_{\text {subl- } \mathrm{H}_{2} \mathrm{O}}=\frac{\rho_{\mathrm{c}}}{\mathrm{S} \mathcal{P}_{\mathrm{H}_{2} \mathrm{O}} \sqrt{\mathrm{m}_{\mathrm{H}_{2} \mathrm{O}} / 2 \pi \mathrm{kT}}}
$$

and similar expressions for $\tau_{\text {subl-CO }}, \tau_{\text {subl- } \mathrm{CO}_{2}}$, and so forth

5. The insolation timescale $\tau_{\odot}$, which concerns the skin of the comet nucleus that is heated by absorption of solar radiation.

$$
\tau_{\odot}=\sqrt{\left(\kappa_{\mathrm{c}} \mathrm{P}_{\mathrm{spin}} / \pi \rho_{\mathrm{c}} \mathrm{c}\right)} \rho_{\mathrm{c}} \mathrm{cT} \frac{4 \pi \mathrm{d}_{\mathrm{H}}^{2}}{\mathrm{~L}_{\odot}(1-\mathcal{A})}
$$

To these, the constant characteristic times of decay of the

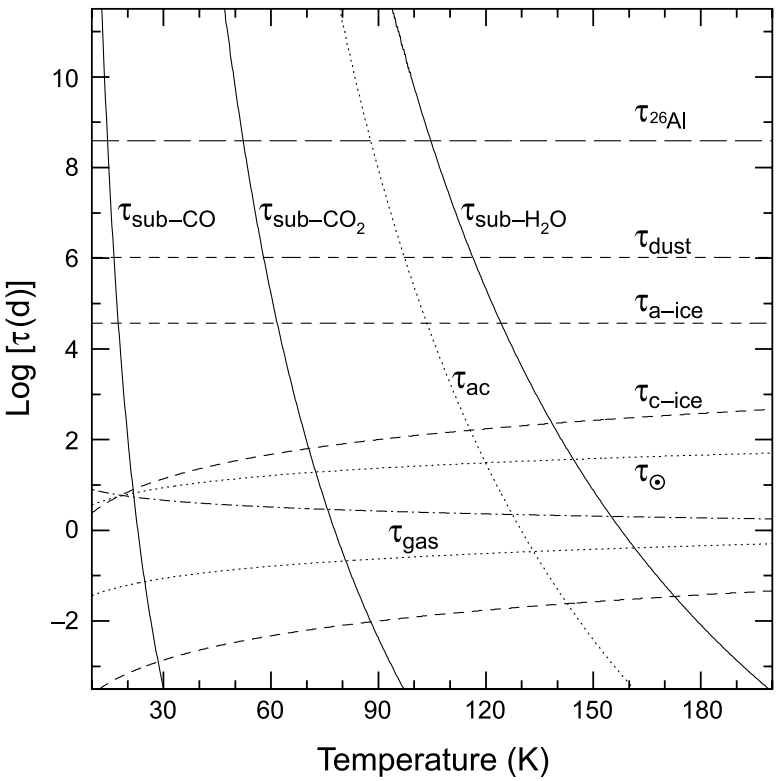

Fig. 4. Timescales of different evolutionary processes (see text) as a function of temperature. The solar timescale is given for distances of 1 and $10 \mathrm{AU}$ (dash-dot lines); the thermal timescale for ice is shown for two depths $(10 \mathrm{~cm}$ and $10 \mathrm{~m})$.

radioactive species may be added; the only relevant one being that of ${ }^{26} \mathrm{Al}$. A comparison of these timescales is shown in Fig. 4, where they are plotted against temperature, assuming an average pore size of $10 \mu \mathrm{m}$ and a porosity of 0.5 , and considering heliocentric distances of 1 and $10 \mathrm{AU}$ and depths of $10 \mathrm{~cm}$ and $10 \mathrm{~m}$ (since the diffusion timescales for heat and gas depend on depth). These timescales will be helpful for understanding the results of numerical calculations presented in the next section.

\section{MODELING RESULTS AND INTERPRETATION: STRUCTURE AND EVOLUTION OF COMET NUCLEI}

\subsection{Input Parameters}

In order to follow the evolution of a comet nucleus structure by means of the equations displayed in section 2 , including the physical processes described in section 3, we still need to specify a number of different types of parameters:

1. Defining parameters. These identify a comet and include orbital parameters (semimajor axis and eccentricity), the nucleus size, given by an average radius, its mass (or bulk density), and its spin period, all of which may be determined observationally.

2. Initial parameters. These are required for the solution of the time-dependent equations and must be guessed. As these equations are not expected to reach equilibrium (steady-state), the initial conditions are not likely to be "forgotten." They include the initial temperature, or tempera- 
ture profile; compositional parameters (mass fractions of the different volatiles and dust); and structural parameters, such as porosity, average pore size or pore size distribution, as well as the nature of the water ice, whether crystalline or amorphous. In fact, one of the main goals of modeling is to determine these properties by comparing their observable consequences to actual observations (see Prialnik, 2002). Regardless of details, initial homogeneity is adopted as a rule.

3. Physical parameters. These are parameters related to the various physical processes discussed in section 3 . They supplement the parameters in the former group and, in principle, should have been determined by them, had the detailed structure of comet nuclei been better understood. Among them are albedo and emissivity, associated with surface properties, as well as thermal conductivity coefficients, tensile strength, and so forth, associated with bulk material properties. These parameters can be determined by laboratory experiments, although caution must be exercised, since laboratory samples are scaled down by orders of magnitude. In situ measurements should eventually provide much more reliable input data.

\subsection{Analytical Considerations: Characteristic Properties of the Comet Nucleus}

Adopting typical values for the physical properties of cometary material in general, simple estimates may be derived for the characteristics of the nucleus structure and evolution, in terms of the defining parameters just mentioned, as shown in Table 5. These provide insight into the nature of comet nuclei, as well as instructive guidelines for building numerical models of their structure and evolution. For example, the skin depth and thermal timescale help in defining an adequate numerical grid, or discretization, in space and time, while the mass loss rate and life span indicate what the size of such a grid should be. The temperature (calculated from equation (1) for the subsolar point) indicates what thermochemical processes are to be expected.

These, however, are only crude estimates. The detailed behavior of comet nuclei is obtained by applying the full set of equations given in section 2 , and including complex input physics, as discussed in section 3 . Since the evolution of nuclei, as already indicated by the crude estimates, is largely determined by their defining parameters, numerical models are mostly applied to individual comets, rather than to comets in general, and even then the results may diverge, as compositional and structural parameters vary. Detailed examples of the application of thermal evolution models to individual comets are given by Meech and Svore ̌r (2004). Typical characteristics of the structure and activity of comet nuclei are discussed in the rest of this section.

\subsection{Surface and Internal Temperatures}

In order to understand the surface temperatures of comet nuclei, we may use the power balance equation at the sur-
TABLE 5. Estimates of characteristic properties of comets.

\begin{tabular}{|c|c|c|}
\hline Property & $\begin{array}{l}\text { Dependence on } \\
\text { Parameters }\end{array}$ & Value \\
\hline Orbital skin depth & $\left(\frac{2 \mathrm{Ka}^{3 / 2}}{G \rho c}\right)^{1 / 2}$ & $18 \mathrm{~m}$ \\
\hline Diurnal skin depth & $\left(\frac{\mathrm{P}_{\text {spin }} \mathrm{K}}{\pi \rho \mathrm{c}}\right)^{1 / 2}$ & $0.1 \mathrm{~m}$ \\
\hline Thermal timescale & $\frac{\mathrm{R}^{2} \rho \mathrm{c}}{\pi^{2} \mathrm{~K}}$ & $8 \times 10^{4} y r$ \\
\hline Insolation per orbit & $\frac{\pi \mathrm{L}_{\odot}}{2 \mathcal{G}} \frac{\mathrm{R}^{2}}{\sqrt{\mathrm{a}\left(1-\mathrm{e}^{2}\right)}}$ & $10^{18} \mathrm{~J}$ \\
\hline Production rate $(\mathrm{ph})$ & $\frac{\mathcal{L}}{4 \mu}\left(\frac{\mathrm{R}}{\mathrm{a}(1-\mathrm{e})}\right)^{2}$ & $1.3 \times 10^{30} / \mathrm{s}$ \\
\hline Erosion per orbit & $\frac{\mathcal{L}}{8 \mathcal{G}} \frac{1}{\rho \sqrt{\mathrm{a}\left(1-\mathrm{e}^{2}\right)}}$ & $1.7 \mathrm{~m}$ \\
\hline Max. temperature & $\frac{4 \pi \mathrm{a}^{2}(1-\mathrm{e})^{2} \mathrm{Q}}{\mathcal{L}}=1$ & $205 \mathrm{~K}$ \\
\hline $\begin{array}{l}\text { Day-night range (ph) } \\
\text { (see equation (79)) }\end{array}$ & $\int_{\mathrm{T}_{\mathrm{n}}}^{\mathrm{T}_{\mathrm{d}}} \sqrt{\frac{\rho \mathrm{cK}}{\pi \mathrm{P}_{\text {spin }}}} \frac{\mathrm{dT}}{\mathrm{QH}}=\frac{1}{2}$ & $23 \mathrm{~K}$ \\
\hline Life time & $\frac{8 \mathcal{G} \mathrm{R} \rho \sqrt{\mathrm{a}\left(1-\mathrm{e}^{2}\right)}}{\mathcal{L}}$ & $3 \times 10^{3} \mathrm{yr}$ \\
\hline
\end{tabular}

Note that $\mathcal{G}=\sqrt{\mathrm{GM}_{\odot}}=1.152 \times 10^{10} \mathrm{~m}^{3 / 2} \mathrm{~s}^{-1} ; \mathcal{L}=\mathrm{L}_{\odot} / \mathcal{H}$; values listed in the last column were obtained using the following parameters: $\mathrm{a}=10 \mathrm{AU}, \mathrm{e}=0.9$, resulting in a perihelion $(\mathrm{ph})$ distance of $1 \mathrm{AU}, \mathrm{R}=5 \mathrm{~km}, \rho=7 \times 10^{2} \mathrm{~kg} / \mathrm{m}^{3}, \mathrm{P}_{\text {spin }}=10 \mathrm{~h}, \mathrm{~K}=0.6 \mathrm{~J} /$ $(\mathrm{m} \mathrm{s} \mathrm{K}), \mathrm{c}=8 \times 10^{2} \mathrm{~J} /(\mathrm{kg} \mathrm{K})$.

face, equation (15), with $-K \nabla T_{R}$ substituted for $F(R)$ on the lefthand side

$$
\frac{(1-\mathcal{A}) \mathrm{L}_{\odot}}{4 \pi \mathrm{d}_{\mathrm{H}}^{2}} \cos \xi=\varepsilon \sigma \mathrm{T}^{4}+\mathrm{K} \nabla \mathrm{T}_{\mathrm{R}}+\mathcal{F} \mathrm{QH}
$$

The incoming solar flux depends mainly on the Sun-comet distance, the rotational state, spin period, scattering properties of the coma (neglected here), and the reflectivity of the surface; thermal reradiation depends on the emissivity; heat transported in and out of the nucleus is a function of the thermal conductivity with all its related parameters; and the energy dissipated in sublimation depends on the composition. For illustration, we choose in the following discussion models of the small, fast-spinning, short-period Comet P46/Wirtanen, the former target of the European mission Rosetta. 
To begin, we distinguish between two extreme cases. The first extreme is to assume that no ices or volatile materials are present at the surface, which then consists of a porous dust layer where gas sublimated in the interior of the nucleus can flow through. Thus the thermal conductivity and matrix structure are the key parameters influencing the surface temperature $\left(T_{s}\right)$. The other extreme is a free sublimation regime, where it is assumed that only water ice is present on the surface. Here the energy used for sublimation of the gas dominates in the power balance equation, at least at heliocentric distances smaller than 2-3 AU. This leads to much lower surface temperatures, as shown in Fig. 5, where results for $T_{s}\left(d_{H}\right)$ at different "comet day" times, obtained from calculations for inactive surfaces (Figs. 5a,b) and for pure ice surfaces (Fig. 5c), are compared. In order to investigate the influence of the thermal conductivity of the mantle on the thermal evolution of the nucleus, we distinguish between a high and a low conductivity mantle. The high value $\mathrm{K}=0.1 \mathrm{Wm}^{-1} \mathrm{~K}^{-1}$ (top panel) is still about a factor of 10 less than the conductivity of typical solid minerals on Earth, so as to account for porosity and for the loose structure of cometary material. For the low conductivity case, a 100-fold smaller value is assumed.

The highest surface temperatures are obtained at noon at the subsolar point; nighttime temperatures are much lower. At perihelion, the difference between the highest temperature ( $370 \mathrm{~K}$ at noon) and the lowest one (about $180 \mathrm{~K}$ at night) is about $190 \mathrm{~K}$, assuming a dust surface. At the intermediate distance of $3 \mathrm{AU}$, the maximum day temperature is about $210 \mathrm{~K}$, while night temperatures are about $140 \mathrm{~K}$. At aphelion the day-night variations are the smallest, about $30 \mathrm{~K}$.

When a much lower thermal conductivity is assumed for the mantle, the difference between day and night temperatures increases significantly. At perihelion, the day-night variation is about $300 \mathrm{~K}\left(\mathrm{~T}_{\max } \approx 380 \mathrm{~K}, \mathrm{~T}_{\min } \approx 80 \mathrm{~K}\right)$ and at aphelion the difference is still quite large, $120 \mathrm{~K}\left(\mathrm{~T}_{\max } \approx\right.$ $180 \mathrm{~K}, \mathrm{~T}_{\min } \approx 60 \mathrm{~K}$ ). The reason is that in the low conductivity case the thermal reradiation power is almost the same as the solar input and the thermal conductivity power is almost null. In the high conductivity case, on the other hand, the power transported by heat conduction into the nucleus is about $10 \%$ of the incoming solar input. At night, stored internal energy is transported from the interior to the surface. This transport, too, is more efficient in case of a high matrix conductivity. Therefore, we obtain smaller temperature differences between day and night and also obtain lower maximum and higher minimum temperatures. The transition from inward to outward heat conduction is not exactly in tune with the insolation power. This transition may be observable: Tracers could be the flux of minor volatiles that depend on the accumulated energy. In the high-conductivity mantle models there is a delay due to the thermal inertia of the material. This delay is vanishingly small in the case of low conductivity. An effect similar to the daynight effect and the associated thermal lag also appears on the much longer timescale of orbital evolution, where pre-
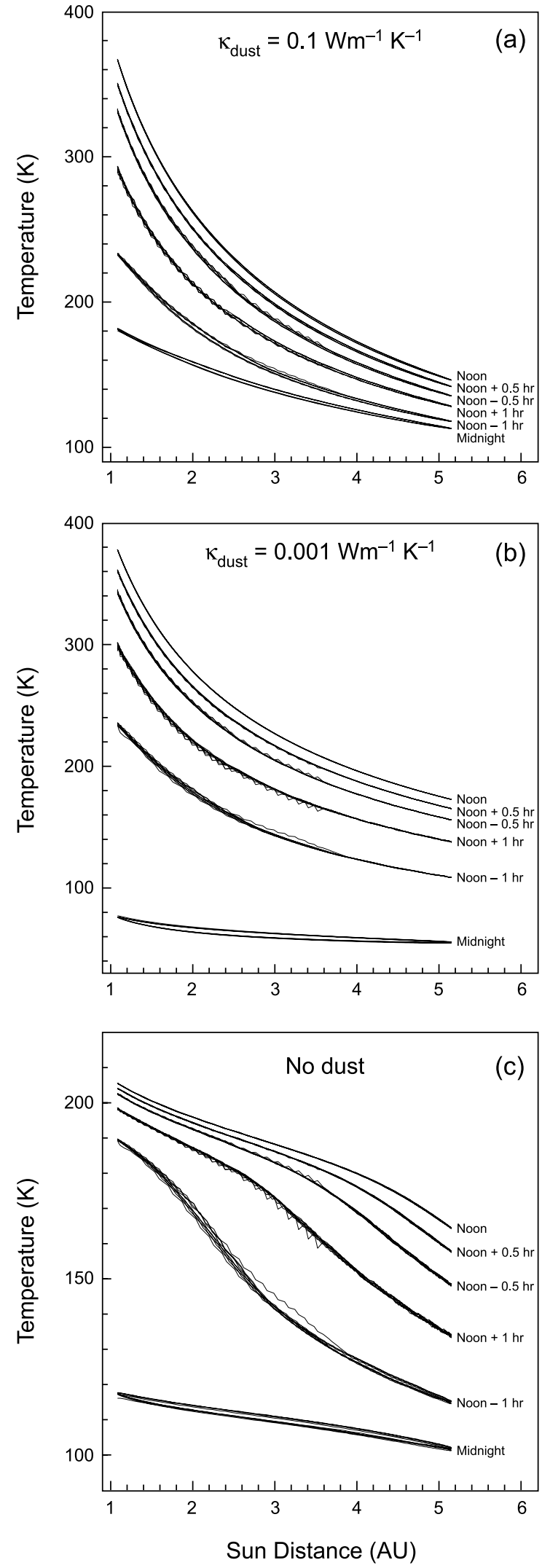

Fig. 5. Surface temperatures as a function of distance from the Sun for different "comet day" times. A comparison is shown between (a),(b) inactive surfaces and (c) pure ice surfaces. Results from model calculations for Comet P46/Wirtanen. 


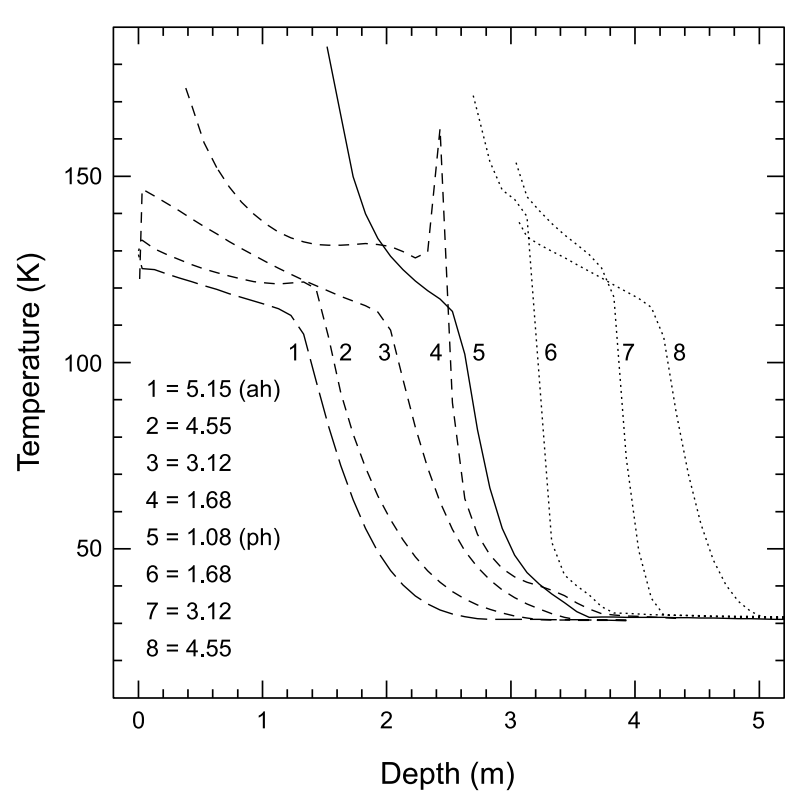

Fig. 6. Temperature profiles in the upper layer of a nucleus model in the orbit of P/Wirtanen at several points along the orbit, pre- and post-perihelion, as marked. The typical steep rise in temperature in curve 4 is due to heat released in crystallization, which proceeds at a fast rate at that point. We note the shift of the surface due to erosion.

perihelion temperatures are typically lower than post-perihelion ones at the same heliocentric distance.

For the free sublimation regime - active areas with no dust mantle on the surface - the results will change significantly. Close to the Sun, at noon, the highest $T_{\mathrm{s}}$ is about $203 \mathrm{~K}$, nearly the free sublimation temperature of water at about $1 \mathrm{AU}$. The lowest $\mathrm{T}_{\mathrm{s}}$ at night is about $115 \mathrm{~K}$, which yields day-night variations of about $90 \mathrm{~K}$ at perihelion. At about $3 \mathrm{AU}$, a maximum of about $190 \mathrm{~K}$ and night minimum of $\sim 105 \mathrm{~K}$ can be expected. At aphelion, the day-night variations are again the smallest and amount to about $65 \mathrm{~K}$ $\left(\mathrm{T}_{\max } \approx 165 \mathrm{~K}, \mathrm{~T}_{\min } \approx 100 \mathrm{~K}\right)$. The large difference between an active and an inactive surface is that in the former most of the insolation power is consumed by water sublimation. Surface temperatures are thus limited by the free sublimation temperature of water ice, a strong energy sink that does not allow temperatures to increase any further. Lower temperatures also reduce the thermal reradiation power, which depends on the temperature to the fourth power.

The heat transported inward serves in part to increase the internal energy of the nucleus, and in part is absorbed in sublimation of volatiles. Heating of the subsurface layers during one revolution is illustrated in Fig. 6 for an amorphous ice nucleus model. The affected region is barely a few meters deep; the layer of temperature inversion at large $d_{H}$ is barely a few centimeters thick. The change in slope of the profile occurs at the boundary between the outer crystalline layer, which is an efficient heat conductor leading to a mild temperature variation with depth, and the inner amorphous part, where conductivity is poor (see section 3.2) and the temperature profile steep.

The diurnal temperature variation may be generally understood and estimated by the following simple argument. The rate of cooling, or the cooling flux $\mathrm{F}_{\text {cool }}(\mathrm{T})$, on the nightside is given by equation (15), setting the insolation rate to zero and assuming a pure ice composition

$$
\mathrm{F}_{\text {cool }}(\mathrm{T})=\varepsilon \sigma \mathrm{T}^{4}+\mathrm{Q}(\mathrm{T}) \mathcal{H}
$$

This is balanced by the heat lost from an outer layer down to a depth equal to the skin depth corresponding to the spin period of the nucleus, $\mathrm{s}=\sqrt{\mathrm{KP}_{\mathrm{spin}} /(\pi \rho \mathrm{c})}$. Thus, over a time interval dt, measured in units of the spin period, the temperature will change by an amount dT given by

$$
\begin{gathered}
\mathrm{F}_{\text {cool }}(\mathrm{T}) \mathrm{dt}=-\rho \mathrm{scd} \mathrm{T}= \\
-\sqrt{\rho \mathrm{\rho c}(\mathrm{T}) \mathrm{K}(\mathrm{T}) /\left(\pi \mathrm{P}_{\text {spin }}\right)} \mathrm{dT}
\end{gathered}
$$

which, integrated over half a spin period, yields

$$
\int_{\mathrm{T}_{\min }}^{\mathrm{T}_{\max }} \frac{\sqrt{\rho c(\mathrm{~T}) \mathrm{K}(\mathrm{T}) /\left(\pi \mathrm{P}_{\text {spin }}\right)}}{\mathrm{F}_{\text {cool }}(\mathrm{T})} \mathrm{dT}=\frac{1}{2}
$$

where $T_{\max }-T_{\min }$ is roughly the temperature difference between the subsolar and antisolar points. The day-night temperature difference as function of $T_{\max }$ is shown in Fig. 7 for three values of the Hertz factor.

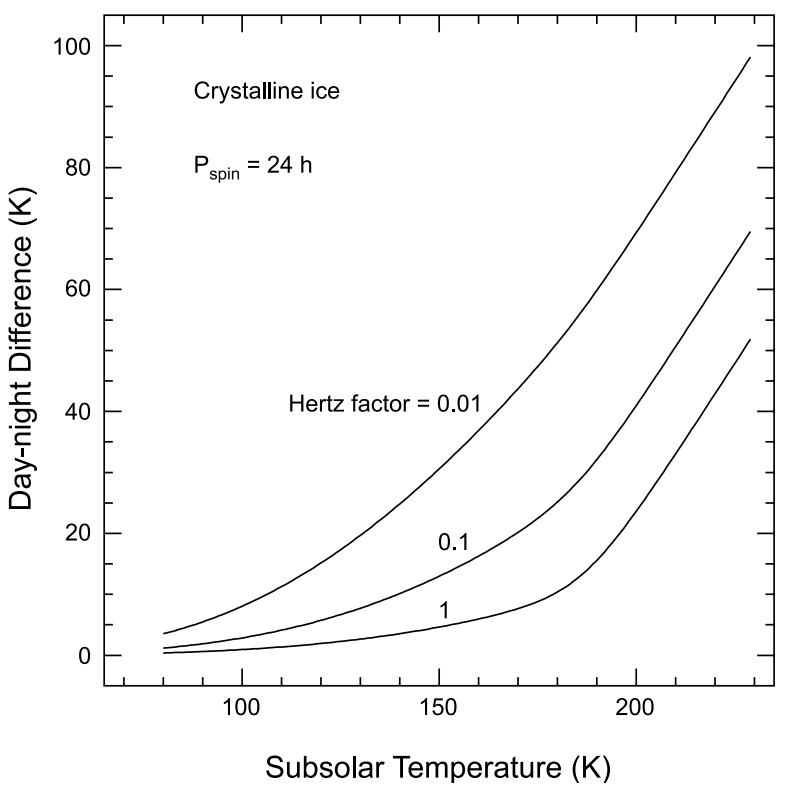

Fig. 7. Analytical estimate for the surface temperature difference between the subsolar and antisolar points for several values of the Hertz factor. 
In terms of timescales (Fig. 4), we note that the timescale of solar heating at $1 \mathrm{AU}$ intersects the sublimation timescales at temperatures $\sim 25 \mathrm{~K}$ for $\mathrm{CO}, \sim 80 \mathrm{~K}$ for $\mathrm{CO}_{2}$, and $\sim 160 \mathrm{~K}$ for $\mathrm{H}_{2} \mathrm{O}$. When these temperatures are attained and if the corresponding ices are found near the surface, the solar energy will be absorbed in sublimation. Consequently, the surface temperature will rise much more slowly. We note that in all cases conduction to the interior is negligibly slow. A steady state will be reached at slightly higher temperatures, when the timescale of gas diffusion for a thin subsurface layer intersects the sublimation timescales: $\sim 30 \mathrm{~K}$ for $\mathrm{CO}, \sim 100 \mathrm{~K}$ for $\mathrm{CO}_{2}$, and $\sim 200 \mathrm{~K}$ for $\mathrm{H}_{2} \mathrm{O}$. These are the expected surface temperatures of comets near $1 \mathrm{AU}$, when the corresponding ices are exposed. If a mixture of ices is present, the temperature will be determined by the most volatile among them.

\subsection{Production and Depletion of Volatiles}

The solar energy flux reaching the nucleus - after being partly scattered or absorbed by the coma - is to some small part reflected (low albedo of the nucleus) and in part reradiated in the IR (high emissivity). A small fraction is transported into the interior of the nucleus by conduction and to a very small degree by radiation in pores. The rest (the bulk at small distances from the Sun) is absorbed at the surface and used to evaporate (sublimate) water ice. The amount of water vapor released depends on the dust cover on the surface. A dust cover a few millimeters thick causes most of the incident energy to be reradiated, leaving only a small fraction for sublimation of water ice. Heat that is conducted into the interior of the porous nucleus may reach ices more volatile than water ice. In a comet nucleus, many different volatile species are expected to be present (see Table 4). If the ice is crystalline, then volatile ices are frozen out as separate phases. As heat diffuses inward, each volatile constituent forms its own sublimation front depending on its enthalpy of vaporization. If amorphous ice is present it will change to crystalline ice, forming an additional front, this time exothermic, for the phase transition. At this front, gases trapped by the amorphous ice will be released. As an ice species evaporates the gas pressure at the sublimation front increases toward its maximum (equilibrium) value at that temperature. The pressure forms a gradient that is negative in the outward direction and positive in the inward direction. This pressure gradient drives the gas flow. The gas flowing outward will diffuse through the comet nucleus and escape through its surface into the coma.

The gas flowing inward will recondense a short distance below the sublimation or crystallization front and release its latent heat. This is an additional heat transport mechanism into the interior, which surpasses advection by flowing gas (Prialnik, 1992; Steiner and Kömle, 1993). It was observed by Benkhoff and Spohn (1991) during the KOSI experiments on cometary ice analogs. Recondensation occurs within a thermal skin depth. The effect is illustrated in Fig. 8.

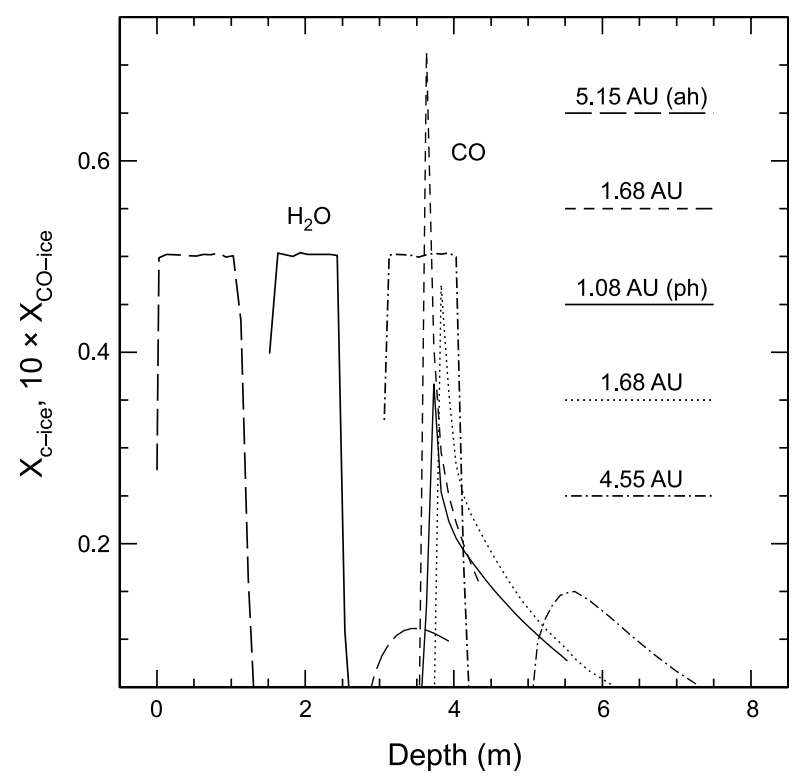

Fig. 8. Mass fraction of crystalline $\mathrm{H}_{2} \mathrm{O}$ ice and of $\mathrm{CO}$ ice (multiplied by 10) in the upper layer of a comet model for the orbit of $\mathrm{P} /$ Wirtanen, at the subsolar point. The initial composition is $\mathrm{X}_{\mathrm{a}}=$ $0.5, f_{\mathrm{CO}}=0.05$, and $\mathrm{X}_{\mathrm{d}}=0.5$. We note the advance of crystallization accompanied by freezing of the $\mathrm{CO}$ gas flowing inward in the cold regions below the crystallization front. The drop of $\mathrm{X}_{\mathrm{c}}$ near the surface is due to sublimation. The model is the same as that of Fig. 6.

Because of heat and gas diffusion, the nucleus will be chemically differentiated in layers. The least-volatile material (dust) will be at the top of the nucleus. It will be followed by a layer of dust and water ice. In the deepest layers we would find dust and all ices, including the most volatile species (such as $\mathrm{CO}$ and $\mathrm{CH}_{4}$ ). At the surface of the nucleus, water and other more volatile ices evaporate, leaving a layer of dust behind. Dust particles entrained by the gas into the coma will heat up in sunlight, and the organic component (hydrocarbon polycondensates) will be vaporized. Polymerized formaldehyde (POM) plays an important role in the dust in producing short-lived formaldehyde gas, which quickly dissociates into $\mathrm{CO}$. The distributed coma source for the $\mathrm{CO}$ must be subtracted from the total $\mathrm{CO}$ release rates in order to obtain production rates resulting from gas released by the nucleus. A major goal of comet research is to determine conditions in the solar nebula based on the chemical composition of comet nuclei. However, the nucleus composition cannot be directly observed and must be deduced from the observed composition of the coma. Taking advantage of new observing technology and the early detection of the very active Comet Hale-Bopp (C/1995 $\mathrm{O} 1)$, researchers were allowed for the first time to determine the coma abundance ratios of different species over a large range of heliocentric distances. The results supported the hypothesis that coma abundances do not reflect in a 
simple way the composition of the nucleus. Abundance ratios of different species may change by factors as large as several hundred, going from heliocentric distances of $r \approx$ $1 \mathrm{AU}$ to $\mathrm{r} \approx 7 \mathrm{AU}$. Thus chemical modeling of the coma coupled to gas-dynamic flow is first required (see Crifo et al., 2004) in order to provide the true, distance-dependent composition of volatiles released from the nucleus. Nucleus models should then be tested against these results along the orbit for as large a range of distances as possible in order to deduce the composition of the nucleus. An example of this procedure, based on the work of Huebner and Benkhoff (1999), is given in Fig. 9, which shows the mixing ratio of CO relative to $\mathrm{H}_{2} \mathrm{O}$ using cubic fits to the release rates obtained by combining observational data from Comet HaleBopp for $\mathrm{H}_{2} \mathrm{O}$ (from $\mathrm{OH}$ ) and $\mathrm{CO}$ covering the spectrum range from radio to UV. The heavy dashed curve is the result of model calculations for a mixture of $35 \%$ amorphous $\mathrm{H}_{2} \mathrm{O}, 7 \% \mathrm{CO}_{2}, 13 \% \mathrm{CO}(50 \%$ trapped in the amorphous ice), and $45 \%$ dust. The $\mathrm{CO}_{2}$ has very little influence on the results. The mixture is close to what one would expect from the condensable component of molecules forming at low temperatures from a mixture of elements with solar abundances. Although the result is below the fit, it must be kept in mind that the $\mathrm{CO}$ from distributed sources has not been subtracted from the observed $\mathrm{CO}$ flux.

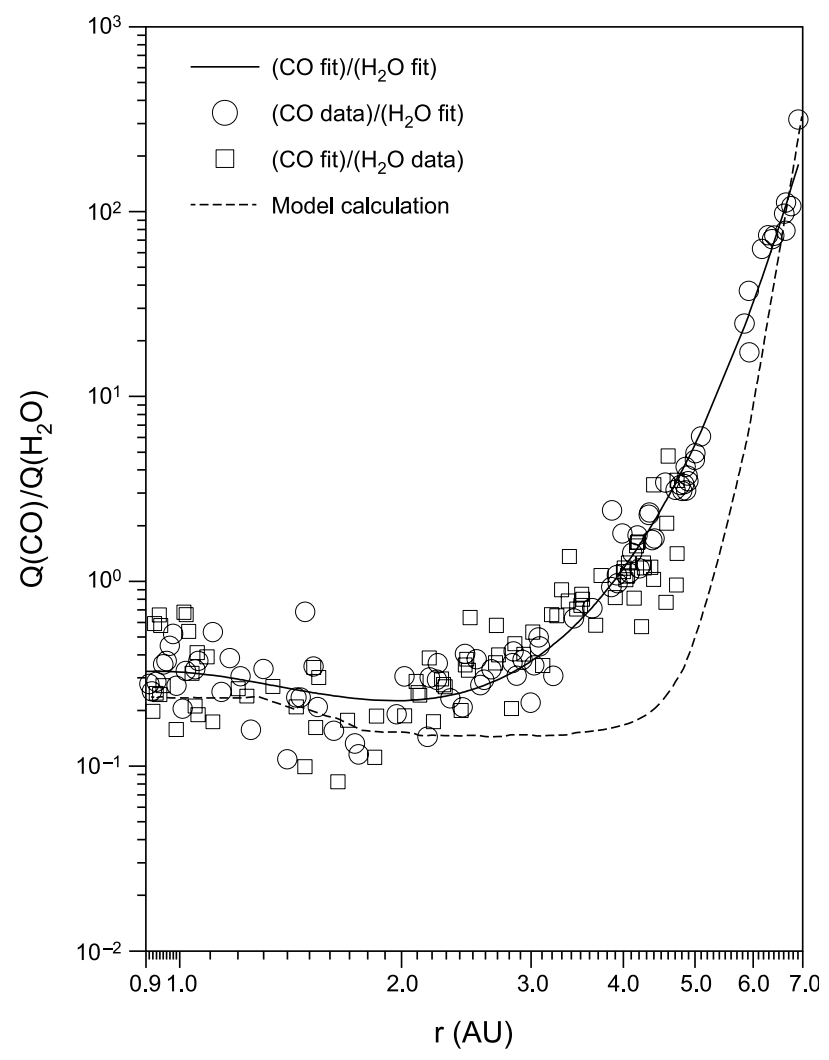

Fig. 9. The mixing ratio of $\mathrm{CO}$ relative to $\mathrm{H}_{2} \mathrm{O}$ using cubic fits to the release rates (see text). From Huebner and Benkhoff (1999).

\subsection{Dust Ejection and Mantle Formation}

The basic process of dust ejection and mantle formation is quite simple: As water and other - more volatile - ices evaporate, the gas flux drags with it dust particles with radii smaller than the critical radius $r_{d}^{*}$ (which varies with temperature), while the larger particles accumulate on the surface, eventually creating an inactive mantle. If at all points of the orbit (that is, for all values of the surface temperature) $r_{d}^{*}(T)<r_{d}^{\max }$, a permanent mantle will form and grow thicker with repeated orbital revolutions. In time, the insulating effect of the mantle will quench sublimation and hence dust entrainment as well. The difficulty in modeling this process is due to the large uncertainties in the parameters involved. Thus, whereas the observed size distribution of dust grains concerns the small particles, it is the large particles that determine the rate of formation of the mantle. Moreover, the physical properties of the mantle may be largely affected by organic material that acts as a glue between dust grains (Kömle et al., 1996). Consequently, one of the main goals of model calculations is to examine the effect of parameter variations. Podolak and Herman (1985) showed that the growth and stability of the mantle is affected by the thermal conductivity, a high conductivity acting as a heat sink. The effect of a variable albedo was studied by Orosei et al. (1995). They showed that dust accumulation and darkening of the surface can cause an increase in the energy absorbed by the nucleus to such an extent as to increase ice sublimation and dust drag to the point of complete removal of the mantle. In such a case the comet would become whiter and colder and buildup of the mantle could start anew, leading to alternating phases of activity and hibernation. The importance of cohesive forces within the refractory material was stressed by Kührt and Keller (1994), who showed that mantles may withstand the vapor pressure building up underneath and thus explain the seemingly permanent inactivity of a large fraction of the nucleus.

Cometary activity declines considerably during the buildup of a dust mantle. A very thin dust layer, on the order of a few centimeters or less, is capable of diminishing the cometary activity by a large factor (e.g., Prialnik and Bar-Nun, 1988; Coradini et al., 1997b; Capria et al., 2001). At the same time, the surface temperature becomes much higher, as we have seen in section 3.7 and Fig. 5. If a dense ice crust builds up below the dust mantle, the activity is quenched to an even higher degree (Prialnik and Mekler, 1991). In these cases the activity is limited to exposed patches of ice. Rickman et al. (1990) show — by numerical simulations of mantle growth - that even stable mantles are sufficiently thin to be broken occasionally by thermal cracks, explosion of gas pockets, or minor collisions, allowing localized activity.

The second goal of models involving dust is to explain the observed dust production rates. Here too the results are largely dependent on unknown parameters. An example of dust ejection from a comet nucleus resulting from dust 


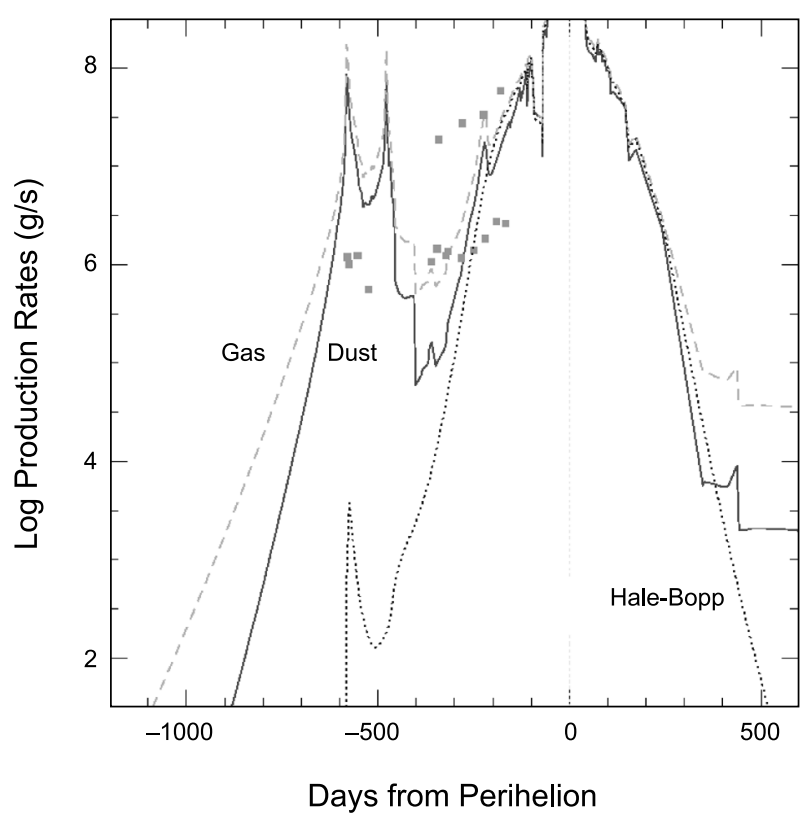

Fig. 10. Dust and gas production rates for a model of Comet Hale-Bopp. The dotted line represents the production rate of $\mathrm{H}_{2} \mathrm{O}$ alone; the dashed line is the total gas flux, including $\mathrm{H}_{2} \mathrm{O}, \mathrm{CO}$, and $\mathrm{CO}_{2}$. The ice/dust mass ratio for the nucleus is 1 . From Prialnik (2002).

carried out from the interior, as well as blown off of the surface, is shown in Fig. 10.

\subsection{Crystallization and Outbursts}

Comets are often found to be active at heliocentric distances far beyond the limit of $\sim 5 \mathrm{AU}$, within which the activity may be explained by sublimation of water ice induced by insolation. Crystallization of amorphous ice has long been recognized as a suitable mechanism for explaining such distant bursts of activity (Patashnik et al., 1974; Smoluchowski, 1981; Espinasse et al., 1991; Weissman, 1991; Prialnik and Bar-Nun, 1992).

Considering the timescales of crystallization, heat conduction and sublimation, we find that at very low temperatures conduction dominates, meaning that heat released by a local source will be efficiently removed. Crystalline ice is a much better heat conductor than amorphous ice and hence heat will flow predominantly to the surface through the growing outer crystalline layer. Thus, as long as the temperature of the outer layer of the nucleus is below the critical temperature where $\tau_{\mathrm{ac}}$ intersects $\tau_{\mathrm{c}-\text { ice }}$ (see Fig. 4), the rate of heating by crystallization will be very slow. As the crystallization rate is much more sensitive to temperature than the conduction rate (of crystalline ice), it will eventually surpass the rate of heat conduction. For example, at a depth of $10 \mathrm{~m}$, the conduction timescale surpasses the crystallization timescale close to $120 \mathrm{~K}$. Crystallization is triggered by some heat source that causes the temperature to rise; when the temperature at the crystallization front reaches the critical temperature $\mathrm{T}_{\mathrm{c}} \sim 110-120 \mathrm{~K}$, the latent heat released at the front causes it to rise still further. The higher temperature, in turn, causes crystallization to proceed even faster and thus a runaway process develops. The rise time and the timescale of outbursts thus triggered should be on the order of $\tau_{c-i c e}\left(T_{c}\right)=\tau_{a c}\left(T_{c}\right)$. According to Fig. 4, it is about 100 days for crystallization for a depth of $10 \mathrm{~m}$ (and it will be $\sim 1$ day for a depth of $1 \mathrm{~m}$ ). This means that fluctuations (and outbursts) at small heliocentric distances should occur on much shorter timescales than at large heliocentric distances. Observations appear to confirm this conclusion.

The competition between $\tau_{\mathrm{ac}}$ and $\tau_{\text {gas }}$ should indicate when an instability is likely to occur. We recall that the crystallization timescale is also the timescale of gas release and pressure buildup (assuming gas is occluded in the amorphous ice), while the diffusion timescale of the gas is also the timescale of pressure relaxation. If $\tau_{\mathrm{ac}}>\tau_{\mathrm{gas}}$, the pressure is released sufficiently rapidly to prevent mechanical instability; however, if $\tau_{\mathrm{gas}} \gg \tau_{\mathrm{ac}}$, gas would accumulate more rapidly than it is removed and large stresses may result from pressure buildup. Thus, if the temperature of amorphous ice at a certain depth exceeds a critical value, it could lead to a state of instability. This situation may be avoided either if the temperature decreases, which is possible if the thermal timescale is sufficiently short, or if the pore size increases, thereby reducing $\tau_{\mathrm{gas}}$. However, according to Fig. 4, the thermal timescales for both amorphous and crystalline ice are longer than $\tau_{\text {gas }}$ by $2-3$ orders of magnitude. Hence only expansion of the pores may arrest the development of an instability, once it occurs. However, the analysis of timescales does not provide clues for the magnitude of the pressure and pressure gradients in relation to the strength of the material, nor to the outcome of unstable conditions. This necessitates detailed numerical computations, and the establishment of an algorithm for treating fracture.

Numerical models of the evolution of cometary nuclei find that crystallization progresses in spurts, their onset, duration, and extent in depth being largely determined both by the structure, composition, and thermal properties of the nucleus and by the comet's orbit (e.g., Herman and Podolak, 1985; Prialnik and Bar-Nun, 1987, 1990; Espinasse et al., 1991, 1993; Tancredi et al., 1994). Crystallization may be initiated by the heat wave propagating inward from the insolated comet surface to the crystalline-amorphous ice boundary, provided that after reaching this boundary, it still carries sufficient energy for significantly raising the local temperature. However, once this has occurred and the boundary has moved deeper into the nucleus, later heat waves originating at the surface will be too weak when reaching the boundary to rekindle crystallization. A quiescent period would thus ensue, until the surface recedes (by sublimation) to a sufficiently short distance from the crystalline-amorphous ice boundary. At this point, a new spurt of crystallization will take place. Since in the meantime the interior temperature of the ice has risen to some extent, crystallization will advance deeper into the nucleus than at the 
previous spurt. This will in turn affect the time span to the next spurt of crystallization, since the rate of surface recession for a given comet is roughly constant (see Table 5). In conclusion, crystallization would appear to be triggered sporadically, preferentially at large heliocentric distances, where comets spend most of their time. This could explain the distant activity — outbursts and possibly splitting — of comets.

The release of gas trapped in the amorphous ice provides the link between crystallization and the eruptive manifestations of comets, of which a few examples will be given below. We have already shown that numerical simulations are based on many simplifying assumptions, and often adopt parameters that are not well known. Hence they should not be expected to accurately reproduce any particular observed outburst. Rather, such simulations should account for the basic characteristics of the observed outbursts.

4.6.1. Distant outbursts of Comet P/Halley. The behavior of Comet P/Halley at large heliocentric distances, beyond $5 \mathrm{AU}$, was characterized by outbursts of various magnitudes; during the most significant one, at $14 \mathrm{AU}$ (West et al., 1991), the total brightness increased by more than $5 \mathrm{mag}$ and an extended coma developed. The outburst subsided on a timescale of months. Klinger and his collaborators (see Espinasse et al., 1991; Weissman, 1991) and Prialnik and Bar-Nun (1992) showed that these features can be explained by ongoing crystallization of amorphous ice in the interior of the porous nucleus, at depths of a few tens of meters. According to this model, enhanced outgassing results from the release of trapped gas during crystallization of the ice. The orbital point where the gas flux reaches its peak was found to be strongly dependent upon the porosity of the comet nucleus. Thus, for example, in the case of a spherical nucleus of porosity $\sim 0.5$ (Prialnik and BarNun, 1990), crystallization is found to occur on the outbound leg of Comet P/Halley's orbit, at heliocentric distances between 5 and $17 \mathrm{AU}$ (depending on the pore size assumed, typical pore sizes being $0.1-10 \mu \mathrm{m})$. Similar results were obtained by Schmitt et al. (1991). The duration of an outburst is the most difficult to predict: Depending on the pore size and on the mechanical properties of the ice, it may vary over three orders of magnitude. A time span of a few months lies within this range and is therefore possible to obtain for a suitable choice of parameters.

4.6.2. Preperihelion activity of 2060 Chiron. Chiron, first classified as an asteroid, was observed to develop a coma at random intervals before it reached perihelion (in 1996) in its 50-year orbit. Marcialis and Buratti (1993) summarized its brightness variations: The first episode of coma formation occurred in 1978, during the middle of the decline in brightness; the second episode, in 1989, when the coma reached vast dimensions, coincided with the maximal brightness. Even near aphelion Chiron underwent a major outburst that lasted several years. Prialnik et al. (1995) were able to obtain a model that agreed remarkably well with the observational data by adopting a composition of $60 \%$ dust and $40 \%$ amorphous ice, occluding a fraction 0.001 of $\mathrm{CO}$ and assuming a low emissivity $(\varepsilon=0.25)$. The optimal parameter combination was found after numerous trials of parameter combinations that proved far less successful. They found that spurts of crystallization started close to aphelion. As a rule, the $\mathrm{CO}$ production rate decreased slightly as the model comet approached the Sun from aphelion. This should explain the puzzling fading of Chiron between 1970 and 1985 (i.e., from $\sim 18$ AU to $\sim 14 \mathrm{AU})$. The model produced the required $\mathrm{CO}$ emission rates, explained by release of trapped gas, and reproduced the estimated surface (color) temperatures at different points of the orbit as derived by Campins et al. (1994). Capria et al. (2000) also explained Chiron's activity by gas trapped in amorphous ice, although they also mentioned the possibility of $\mathrm{CO}$ ice close to the surface, which would imply that Chiron has been inserted into its present orbit only recently (cf. Fanale and Salvail, 1997).

4.6.3. Erratic activity of Comet Schwassmann-Wachmann 1 (SW1). The orbit of Comet SW1 is nearly circular and confined between the orbits of Jupiter and Saturn. Despite the fact that at such heliocentric distances the sublimation of $\mathrm{H}_{2} \mathrm{O}$ ice is negligible, this comet exhibits irregular activity - unpredictable changes in its lightcurve. Froeschle et al. (1983) suggested that this might be associated with crystallization of amorphous ice. This suggestion was further strengthened by the detection of $\mathrm{CO}$ released by the comet (Senay and Jewitt, 1994; Crovisier et al., 1995), since although SW1 is too distant for $\mathrm{H}_{2} \mathrm{O}$ ice sublimation, its surface is too hot for the survival of $\mathrm{CO}$ ice. Subsequently, Klinger et al. (1996) showed by model calculations that the $\mathrm{CO}$ production pattern can be explained and simulated by gas trapped in the amorphous ice and released from the ice upon crystallization. The chaotic behavior results from the highly nonlinear temperature dependence of the processes involved.

4.6.4. Distant activity of Comet Hale-Bopp. Comet Hale-Bopp (C/1995 O1) was characterized by an unusually bright coma at a distance of about 7 AU from the Sun. Observations performed by Jewitt et al. (1996) detected a very large flux of $\mathrm{CO}$ molecules, which increased dramatically. Such brightening is unlikely to have resulted from surface (or subsurface) sublimation of $\mathrm{CO}$ ice in response to insolation. In any case, $\mathrm{CO}$ ice should have been depleted much earlier in the orbit, since at $7 \mathrm{AU}$ the surface temperature is already above $100 \mathrm{~K}$, considerably higher than the sublimation temperature of $\mathrm{CO}$. In this case as well the unusual activity could be explained on the basis of crystallization and release of occluded $\mathrm{CO}$ accompanied by ejection of dust entrained by the gas (Prialnik, 1999, 2002; Capria et al., 2002).

\subsection{Early Evolution of Comets: Effect of Radioactivity}

Formation of comets, like star formation, is still an object of study and thermal evolution during formation has barely been considered (Merk, 2003). But attempts to estimate the possible effect of radioactive heating on young comet nuclei have been made in a number of different stud- 
ies under different assumptions and approximations [see Prialnik and Podolak (1995) and references therein, and more recently, De Sanctis et al. (2001) and Choi et al. (2002) and references therein]. There is general agreement that the long-lived radionuclides should have no or little effect on objects below about $50 \mathrm{~km}$ in radius; thus ${ }^{26} \mathrm{Al}$ is considered as the energy source. Using again Fig. 4 as a guide, we find by extrapolation that at a depth of $1 \mathrm{~km}$ the thermal timescale of amorphous ice becomes comparable to the decay time of ${ }^{26} \mathrm{Al}$, meaning that the ice may barely be heated. It will certainly be heated at larger depths, a few kilometers and beyond. There, eventually, the internal temperature will become sufficiently high for crystallization to set in, providing an additional internal heat source (Podolak and Prialnik, 1997). At the same time, however, the thermal timescale will decrease, crystalline ice being a much better heat conductor than amorphous ice. In addition, if the nucleus is sufficiently porous, the gases released upon crystallization will be able to escape to the outer regions of the nucleus and will carry the heat away efficiently. Hence, only in still larger comet nuclei (beyond $10 \mathrm{~km}$ ) will the internal temperature continue to rise.

If the internal temperature becomes such that the timescale of sublimation is shorter than the timescale of radiogenic heat release, then most of the released energy will be absorbed in sublimation of ice from the pore walls, starting with the most volatile species. If, in addition, the radius is such that the timescale of gas (vapor) diffusion is lower than the timescale of sublimation, then sublimation will consume the radiogenic heat so long as there is ice, since the vapor will be efficiently removed. A steady state will develop, without further heating of the ice matrix. Regarding $\mathrm{H}_{2} \mathrm{O}$, it is worth mentioning that the temperature of such a steady state would be considerably lower than the melting temperature of ice. On the other hand, if the porosity of the ice is very low and the average pore size very small, $\tau_{\text {gas }}$ may become sufficiently high for gas removal to become inefficient. In such cases the internal temperature may rise to the melting point of ice (cf. Yaboushita, 1993; Podolak and Prialnik, 2000).

Calculations of the long-term evolution of comets far from the Sun under the influence of radioactive heating show that the internal temperatures attained may be sufficiently high for comets to have become depleted of volatiles that sublimate below $\sim 40-50 \mathrm{~K}$, initially included as ices (De Sanctis et al., 2001; Choi et al., 2002). Less-volatile species may have been partly lost as well. Observation of such volatiles in comets suggests that they originate from amorphous $\mathrm{H}_{2} \mathrm{O}$ ice undergoing crystallization. This means that, despite radioactive heating, a substantial fraction of the $\mathrm{H}_{2} \mathrm{O}$ ice has retained its pristine form, i.e., the innermost region is crystalline, and the outer region is composed of amorphous ice. Figure 11 shows the relative radius of the inner part of the nucleus that has crystallized during early evolution, as a function of the comet's radius and distance from the Sun (Merk, 2003).

Although much of the released gas will escape the nucleus entirely, some will become trapped in the cold outer

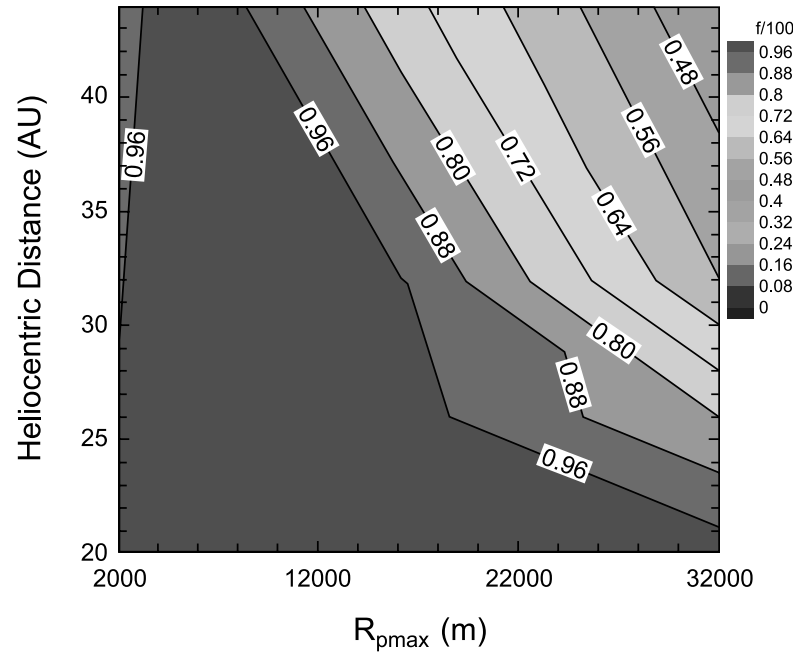

Fig. 11. Contour plots of the relative radius $r / R$ up to which the nucleus core crystallizes due to radiogenic heating during formation by accretion. From Merk (2003).

layers of the nucleus. This will reduce the porosity in those layers and enrich them in condensed volatiles. As the comet nears the Sun and the outer layers heat up, these gases will be released and the comet will show enhanced activity.

\section{CONCLUSIONS AND DIRECTIONS FOR FUTURE WORK}

\subsection{General Conclusions}

In spite of the sparse information regarding the cometary interior, the complexity of the processes that may take place within them, and the uncertainties involved, the general conclusion that emerges from simulations of the evolution of comet nuclei is that, essentially, a nucleus model of porous, grainy material, composed of gas-laden amorphous ice and dust, is capable of reproducing the activity pattern of comets. Three types of cometary activity, all associated with the flow of volatiles through and out of a porous nucleus, are identified. They have observable outward manifestations on the one hand, and lasting effects on the structure of the nucleus on the other.

1. Sublimation of volatiles from the pore walls and the subsequent flow of vapor is the source of gas for the coma and tail, but may also lead to the formation of a dense ice crust below the surface of the nucleus. Gases flowing to the interior may refreeze when reaching sufficiently cold regions, at depths correlated with the volatility of the gas. The resulting effect is a stratified nucleus configuration.

2. Crystallization of amorphous ice, accompanied by the release of heat as well as trapped gases, may account for cometary outbursts and may also result in fracture of the porous material.

3. Drag of dust grains by the flowing gas leads to ejection of the small particles seen in the dust coma and tail, while accumulation of the large particles on the surface of 


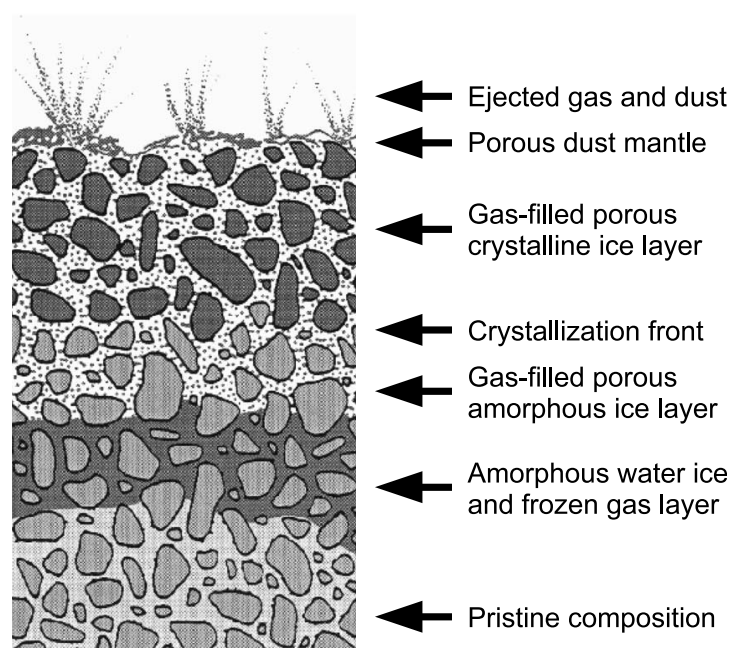

Fig. 12. Schematic layered structure of a cometary nucleus (arbitrary scales). From Prialnik (1999).

the nucleus may lead to the formation of a sealing dust mantle that would turn the comet into an asteroid-like object.

In conclusion, the thermal evolution and activity pattern of a porous comet nucleus differs significantly from the old view of a solid icy body that is mainly controlled by sublimation from the surface in response to solar heating. The structure that emerges is shown schematically in Fig. 12.

The thermal evolution of comet nuclei may be divided into two phases: a long phase - of the order of the solar system's age - spent at large distances from the Sun (in the Oort cloud or the Kuiper belt), and a second, much shorter phase, spent in orbit around the Sun within the planetary system. There is, of course, an intermediate, transient phase during which a periodic comet is gradually perturbed into its final, steady orbit. The notion that the thermal evolution process really begins when a comet enters the second phase of its life, becoming a "new" comet, is beginning to be doubted. New comets, which have often been described as pristine objects that have undergone no (or little) alteration during their lifetime in the distant outskirts of the solar system, are now suspected to have been heated to the point of melting of the $\mathrm{H}_{2} \mathrm{O}$ ice. Nevertheless, they are still believed to constitute a source of solar nebula material. Much of the fascination and interest comets have aroused was due to the clues they were believed to hold to the formation of the solar system. This may still be true, at least for a fraction of comets, or for a fraction of every comet. In addition, comets are now invoked to explain the formation of life.

\subsection{Required Input Data from Observations and Experiments}

The success of the thermal evolution theory just described in explaining the structure and activity of comet nuclei is hindered by the huge lack of information regard- ing crucial (or critical) parameters. As a result, explanations for observed behavior may be ambiguous; i.e., different parameter combinations, within the same model, may lead to similar results. Consequently, additional input is required both from laboratory studies and from observations.

The input required from laboratory studies includes (1) pressure curves at low temperatures, (2) latent heat measurement, (3) thermal conductivity of mixtures, and (4) sublimation studies of mixtures. From observations we need more information on dynamical properties (spin axis, rotation period, orientation, and shape of nucleus). It would be interesting to determine and understand whether a potato, rather than spherical, shape is typical of small bodies of negligible self-gravity. Upcoming in situ measurements should provide information about the porous structure porosity and pore size - as well as strength. The interplay among the different methods of research applied to cometary nuclei is illustrated in Fig. 13.

\subsection{Where Do We Go from Here?}

In the course of this review we have mentioned a rather long list of assumptions that are common to most theoretical studies to date. Simplifying assumptions are justified when a theory is still young and ridden by uncertainties. Now that it has matured, we may safely enter the next stage, where more sophisticated methods and models should be developed. We suggest a few below, following the order of the review's sections. In some cases, first steps have already been taken.

5.3.1. Numerical methods. (1) Use of adaptive grid methods for dealing with receding surfaces during late evolution, as well as growing mass during very early stages. (2) Development of full-scale 3-D models that allow for lateral flow both of heat and of gas. (3) Inclusion of boundary conditions accounting for the nucleus-coma interaction. (4) Implementation of modern methods for the simultaneous solution of a multiple component nucleus.

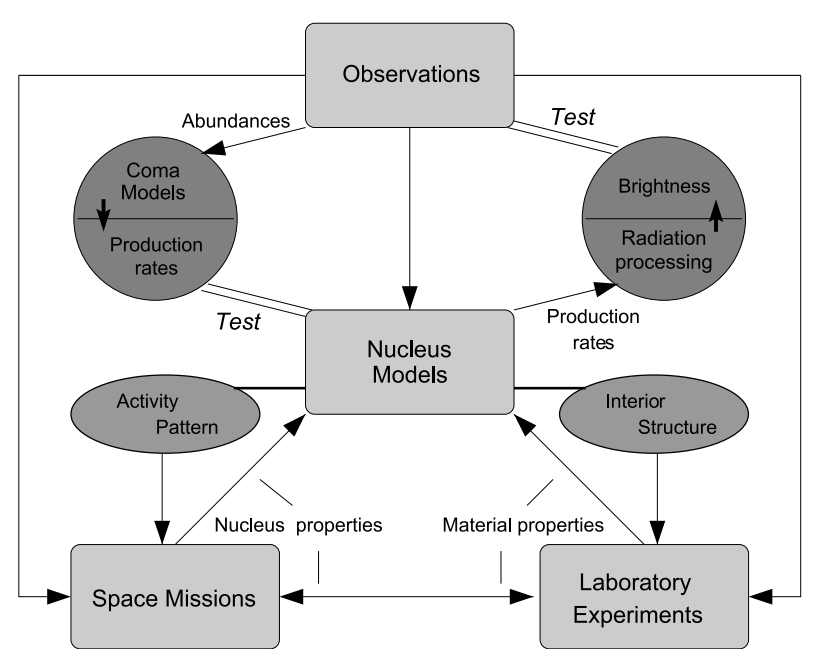

Fig. 13. The role of nucleus models in the coordinated study of comets. 
5.3.2. Physical processes. (1) Coupling between gas phases and rigorous treatment of mixtures. (2) Construction of models for fracture and for crack propagation. (3) Treatment of surface properties, such as irregularities, shadowing, and mixed thermal properties, as well as radiative transfer in the outermost porous layer (e.g., Davidsson and Skorov, 2002a,b). (4) Modeling of the material structure of the dust mantle on the nucleus surface.

5.3.3. Modeling the evolution of comet nuclei. (1) Modeling comet formation by accretion. (2) Long-term evolution over the age of the solar system, considering potential gravitational interactions and orbital evolution. (3) Modeling comet $\Leftrightarrow$ asteroid transition (e.g., Coradini et al., 1997a). (4) Modeling nucleus shape evolution as a result of uneven ablation.

The purpose of modeling comet nuclei is not to predict their behavior based on an initial set of parameters. Given the large number of parameters and their wide range of possible values, predictions may be misleading. Rather, the true purpose of modeling is to reproduce the observed cometary behavior, in order to deduce internal properties of comet nuclei that are inaccessible to observation. The closer the numerical simulations are to observed reality, the more reliable will be our inferences on the elusive nature of comet nuclei and the clues they hold to the understanding of the solar system's beginnings - perhaps to the beginning of life as well. In the words of Isaac Newton: "I suspect that the spirit which is the smallest but most subtle and most excellent part of our air, and which is required for the life of all things, comes chiefly from comets" [Principia, Book 3, Proposition 41, 1687 (translation from Cohen and Whitman, 1999].

\section{REFERENCES}

Arakawa M., Higa M., Leliwa-Kopystyński J., and Maeno N. (2000) Impact cratering of granular mixture targets made of $\mathrm{H}_{2} \mathrm{O}$ ice $-\mathrm{CO}_{2}$ ice-pyrophylite. Planet. Space Sci., 48, 14371446.

Bar-Nun A. and Owen T. (1998) Trapping of gases in water ice. In Solar System Ices (B. Schmitt et al., eds.), pp. 353-366. Kluwer, Dordrecht.

Bar-Nun A., Dror J., Kochavi E., and Laufer D. (1987) Amorphous water ice and its ability to trap gases. Phys. Rev. B, 35, 2427-2435.

Benkhoff J. (1999) Energy balance and the gas flux from the surface of Comet 46P/Wirtanen. Planet. Space Sci., 47, 735-744.

Benkhoff J. (2002) The emission of gas and dust from comets. Adv. Space Res., 29, 1177-1186.

Benkhoff J. and Boice D. C. (1996) Modeling the thermal properties and the gas flux from a porous, ice body in the orbit of P/Wirtanen. Planet. Space Sci., 44, 665-674.

Benkhoff J. and Huebner W. F. (1995) Influence of the vapor flux on temperature, density, and abundance distribution in a multicomponent, porous ice body. Icarus, 114, 348-354.

Benkhoff J. and Spohn T. (1991) Thermal histories of the KOSI samples. Geophys. Res. Lett., 18, 261-264.

Blake D., Allamadolla L., Sandford S., Hudgins D., and Freund F. (1991) Clathrate hydrate formation in amorphous cometary ice analogs in vacuo. Science, $245,58-551$.

Bouziani N. and Fanale F. P. (1998) Physical chemistry of a heterogeneous medium: Transport processes in comet nuclei. Astrophys. J., 499, 463-474.

Brailsford A. and Major K. G. (1964) The thermal conductivity of aggregates of several phases, including porous materials. $\mathrm{Br}$. J. Appl. Phys., 15, 313.

Brin G. D. and Mendis D. A. (1979) Dust release and mantle development in comets. Astrophys. J., 229, 402-408.

Campins H., Telesco C. M., Osip D. J., Rieke G. H., Rieke M. J., and Schulz B. (1994) The color temperature of (2060) Chiron: A warm and small nucleus. Astron. J., 108, 2318-2322.

Capria M. T., Coradini A., De Sanctis M. C., and Orosei R. (2000) Chiron activity and thermal evolution. Astron. J., 119, 31123118.

Capria M. T., Coradini A., De Sanctis M. C., and Bleckai M. I. (2001) P/Wirtanen thermal evolution: Effects due to the presence of an organic component in the refractory material. Planet. Space Sci., 49, 907-918.

Capria M. T., Coradini A., and De Sanctis M. C. (2002) C/1995 O1 Hale-Bopp: Short and long distance activity from a theoretical model. Earth Moon Planets, 90, 217-225.

Choi Y.-J., Cohen M., Merk R., and Prialnik D. (2002) Long-term evolution of objects in the Kuiper belt zone - effects of insolation and radiogenic heating. Icarus, 160, 300-312.

Cohen I. B. and Whitman A. (1999) Isaac Newton - The Principia. Univ. of California, Berkeley. 974 pp.

Cohen M., Prialnik D., and Podolak M. (2003) A quasi-3D model for the evolution of shape and temperature distribution of comet nuclei - application to Comet 46P/Wirtanen. New Astron., 8, 179-189.

Cook R. F. (1989) Effective-medium theory for the fracture of fractal porous media. Phys. Rev. B, 39, 2811-2814.

Coradini A., Capaccioni F., Capria M. T., De Sanctis M. C., Espinassse S., Orosei R., and Salomone M.(1997a) Transition elements between comets and asteroids. I. Thermal evolution models. Icarus, 129, 317-336.

Coradini A., Capaccioni F., Capria M. T., De Sanctis M. C., Espinassse S., Orosei R., and Salomone M. (1997b) Transition elements between comets and asteroids. II. From the Kuiper belt to NEO orbits. Icarus, 129, 337-347.

Cowan J. J. and A'Hearn M. F. (1979) Vaporization of comet nuclei - Light curves and life times. Moon and Planets, 21, $155-171$.

Crifo J. F. and Rodionov A. V. (1997) The dependence of the circumnuclear coma structure on the properties of the nucleus. I. Comparison between homogeneous and inhomogeneous nucleus spherical nucleus with application to P/Wirtanen. Icarus, 127, 319-353.

Crifo J. F., Fulle M., Kömle N. I., and Szeg K. (2004) Nucleuscoma structural relationships: Lessons from physical models. In Comets II (M. C. Festou et al., eds.), this volume. Univ. of Arizona, Tucson.

Crovisier J., Biver N., Bockelée-Morvan D., Colom P., Jorda L., Lellouch E., Paubert G., and Despois D. (1995) Carbon monoxide outgassing from Comet Schwassmann-Wachmann 1. Icarus, 115, 213-216.

Davidsson B. J. R. (1999) Tidal splitting and rotational breakup of solid spheres. Icarus, 142, 525-535.

Davidsson B. J. R. (2001) Tidal splitting and rotational breakup of solid biaxial ellipsoids. Icarus, 149, 375-383.

Davidsson B. J. R. and Skorov Yu. V. (2002a) On the light-absorb- 
ing surface layer of cometary nuclei: I. Radiative transfer. Icarus, 156, 223-248.

Davidsson B. J. R. and Skorov Yu. V. (2002b) On the light-absorbing surface layer of cometary nuclei: II. Thermal modeling. Icarus, 159, 239-258.

De Sanctis M. C., Capria M. T., and Coradini A. (2001) Thermal evolution and differentiation of Kuiper belt objects. Astron. J., 121, 2792-2799.

Eluszkiewicz J., Leliwa-Kopystyński J., and Kossacki K. J. (1998) Metamorphism of solar system ices. In Solar System Ices (B. Schmitt et al., eds.), pp. 119-138. Kluwer, Dordrecht.

Enzian A., Cabot H., and Klinger J. (1997) A 2 1/2 D thermodynamic model of cometary nuclei. Astron. Astrophys., 31, 9951006.

Enzian A., Klinger J., Schwehm G., and Weissman P. R. (1999) Temperature and gas production distribution on the surface of a spherical model nucleus in the orbit of 46P/Wirtanen. Icarus, 138, 74-84.

Espinasse S., Klinger J., Ritz C., and Schmitt B. (1991) Modeling of the thermal behavior and of the chemical differentiation of cometary nuclei. Icarus, 92, 350-365.

Espinasse S., Coradini A., Capria M. T., Capaccioni F., Orosei R., Salomone M., and Federico C. (1993) Thermal evolution and differentiation of a short period comet. Planet. Space Sci., 41, 409-427.

Fanale F. P. and Salvail J. R. (1984) An idealized short period comet model: Surface insolation, flux, dust flux, and mantle evolution. Icarus, 60, 476-511.

Fanale F. P. and Salvail J. R. (1987) The loss and depth of $\mathrm{CO}_{2}$ ice in comet nuclei. Icarus, 72, 535-554.

Fanale F. P. and Salvail J. R. (1990) The influence of CO ice on the activity and near-surface differentiation of comet nuclei. Icarus, 84, 403-413.

Fanale F. P. and Salvail J. R. (1997) The cometary activity of Chiron: A stratigraphic model. Icarus, 125, 397-405.

Froeschlé Cl., Klinger J., and Rickman H. (1983) Thermal models for the nucleus of Comet P/Schwassmann-Wachmann 1. In Asteroids, Comets, Meteors (A85-26851 11-89), pp. 215-224.

Greenberg J. M., Mizutani H., and Yamamoto T. (1995) A new derivation of the tensile strength of cometary nuclei: Application to Comet Shoemaker-Levy 9. Astron. Astrophys., 295, L35-L38.

Grün E., Gebhard J., Bar-Nun A., Benkhoff J., Düren H., Eich G., Hische R., Huebner W. F., Keller H. U., and Klees G. (1993) Development of a dust mantle on the surface of an insolated ice-dust mixture: Results of the KOSI-9 experiment. J. Geophys. Res., 98, 15091-15104.

Gutiérrez P. J., Ortiz J. L., Rodrigo R., and Lopez-Moreno J. J. (2000) A study of water production and temperature of rotating irregularly shaped cometary nuclei. Astron. Astrophys., 355, 809-817.

Haruyama J., Yamamoto T., Mizutani H., and Greenberg J. M. (1993) Thermal history of comets during residence in the Oort cloud: Effect of radiogenic heating in combination with the very low thermal conductivity of amorphous ice. J. Geophys. Res., 98, 15079-15090.

Herman G. and Podolak M. (1985) Numerical simulations of comet nuclei: I. Water ice comets. Icarus, 61, 252-266.

Herman G. and Weissman P. R. (1987) Numerical simulation of cometary nuclei III. Internal temperatures of cometary nuclei. Icarus, 69, 314-328.

Horanyi M., Gombosi T. I., Cravens T. E., Körösmezey A.,
Kecskeméty K., Nagy A. F., and Szegö K. (1984) The friable sponge model of a cometary nucleus. Astrophys. J., 278, 449455.

Huebner W. F. and Benkhoff J. (1999) From coma abundances to nucleus composition. In Proceedings of the ISSI-Workshop: The Origin and Composition of Cometary Material (K. Altweg et al., eds.). Space Sci. Rev., 90, 117-130.

Huebner W. F., Benkhoff J., Capria M. T., Coradini A., De Sanctis C., Enzian A., Orosei R., and Prialnik D. (1999) Results from the comet nucleus model team at the international space science institute, Bern, Switzerland. Adv. Space Res., 23, 1283 1298.

Irvine W. M., Leschine S. B., and Schloerb F. P. (1980) Thermal history, chemical composition and relationship of comets to the origin of life. Nature, 283, 748-749.

Jenniskens P. and Blake D. F. (1994) Structural transitions in amorphous water ice and astrophysical implications. Science, 265, 753-756.

Jewitt D., Senay M., and Matthews H. (1996) Observations of carbon monoxide in Comet Hale-Bopp. Science, 271, 11101113.

Julian W. H., Samarasinha N. H., and Belton M. J. S. (2000) Thermal structure of cometary active regions: Comet 1P/Halley. Icarus, 144, 160-171.

Kaponen A., Kataja M., and Timonen J. (1997) Permeability and effective porosity of porous media. Phys. Rev. E, 56, 33193325.

Klinger J. (1980) Influence of a phase transition of ice on the heat and mass balance of comets. Science, 209, 271-272.

Klinger J. (1981) Some consequences of a phase transition of water ice on the heat balance of comet nuclei. Icarus, 47, 320324.

Klinger J., Espinasse S., and Schmidt B. (1989) Some considerations on cohesive forces in sun-grazing comets. In Proceedings of an International Workshop on Physics and Mechanics of Cometary Materials (J. Hunt and T. D. Guyeme, eds.), pp. 197-200. ESA SP-302, Noordwijk, The Netherlands.

Klinger J., Levasseur-Regourd A. C., Bouziani N., and Enzian A. (1996) Towards a model of cometary nuclei for engineering studies for future space craft missions to comets. Planet. Space Sci., 44, 637-653.

Kochan H., Roessler K., Ratke L., Heyl M., Hellman H., and Schwehm G. (1989) Crustal strength of different model comet materials. Proceedings of an International Workshop on Physics and Mechanics of Cometary Materials (J. Hunt and T. D. Guyeme, eds.), pp. 115-119. ESA SP-302, Noordwijk, The Netherlands.

Kömle N. I., Kargl G., Thiel K., and Seiferlin K. (1996) Thermal properties of cometary ices and sublimation residua including organics. Planet. Space Sci., 44, 675-689.

Kossacki K. J., Szutowicz S., and Leliwa-Kopystyński J. (1999) Comet 46P/Wirtanen: Evolution of the subsurface layer. Icarus, 142, 202-218.

Kouchi A., Greenberg J. M., Yamamoto T., and Mukai T. (1992) Extremely low thermal conductivity of amorphous ice: Relevance to comet evolution. Astrophys. J. Lett., 388, L73-L76.

Kouchi A., Yamamoto T., Kozasa T., Koruda T., and Greenberg J. M. (1994) Conditions for condensation and preservation of amorphous ice and crystallinity of astrophysical ices. Astron. Astrophys., 290, 1009-1018.

Kührt E. (1984) Temperature profiles and thermal stresses in cometary nuclei. Icarus, 60, 512-521. 
Kührt E. and Keller H. U. (1994) The formation of cometary surface crusts. Icarus, 109, 121-132.

Lee T., Papanastassiou D., and Wasserburg G. J. (1976) Demonstration of ${ }^{26} \mathrm{Mg}$ excess in Allende and evidence for ${ }^{26} \mathrm{Al}$. Geophys. Res. Lett., 3, 109-112.

Lide D. R. (2003) CRC Handbook of Chemistry and Physics, 84th edition. CRC Press, Boca Raton, Florida. 2616 pp.

Lugmair G. W. and Shukolyukov A. (2001) Early solar system events and timescales. Meteoritics \& Planet. Sci., 36, 10171026.

MacPherson G. J., Davis A. M., and Zinner E. K. (1995) The distribution of aluminum-26 in the early solar system - A reappraisal. Meteoritics, 30, 365-386.

Mahoney W. A., Ling J. C., Wheaton Wm. A., and Jacobson A. S. (1984) Heao 3 discovery of ${ }^{26} \mathrm{Al}$ in the interstellar medium. Astrophys. J., 286, 578-585.

Marcialis R. L. and Buratti B. J. (1993) CCD photometry of 2060 Chiron in 1985 and 1991. Icarus, 104, 234-243.

Maxwell J. C. (1873) A Treatise on Electricity and Magnetism. Clarendon, Oxford.

Meech K. J. and Svoreň J. (2004) Physical and chemical evolution of cometary nuclei. In Comets II (M. C. Festou et al., eds.), this volume. Univ. of Arizona, Tucson.

Mekler Y. and Podolak M. (1994) Formation of amorphous ice in the protoplanetary nebula. Planet. Space Sci., 42, 865-870.

Mekler Y., Prialnik D., and Podolak M. (1990) Evaporation from a porous cometary nucleus. Astrophys. J., 356, 682-686.

Mendis D. A. and Brin G. D. (1977) Monochromatic brightness variations of comets. II - Core-mantle model. Moon, 17, 359372.

Merk R. (2003) Thermodynamics and accretino of asteroids, comets, and Kuiper Belt Objects - A computer simulation study in plaentary physics. Ph.D. thesis, Univ. of Münster. 143 pp.

Merk R., Breuer D., and Spohn T. (2002) Numerical modeling of ${ }^{26} \mathrm{Al}$-induced melting of asteroids considering accretion. Icarus, 159, 183-191.

Morley A. (1954) Strength of Materials, 11th edition. Longmans, London. 532 pp.

Öpik E. J. (1958) Physics of Meteor Flight in the Atmosphere. Interscience, New York. 174 pp.

Orosei R., Capaccioni F., Capria M. T., Coradini A., Espinasse S., Federico C., Salomone M., and Schwehm G. H. (1995) Gas and dust emission from a dusty porous comet. Astron. Astrophys., 301, 613-627.

Patashnick H., Rupprecht G., and Schuerman D. W. (1974) Energy source for comet outbursts. Nature, 250, 313-314.

Podolak M. and Herman G. (1985) Numerical simulations of comet nuclei II. The effect of the dust mantle. Icarus, 61, 267277.

Podolak M. and Prialnik D. (1996) Models of the structure and evolution of Comet P/Wirtanen. Planet. Space Sci., 44, 655664.

Podolak M. and Prialnik D. (1997) ${ }^{26} \mathrm{Al}$ and liquid water environments in comet. In Comets and the Origin of Life (P. Thomas et al., eds.), pp. 259-272. Springer-Verlag, New York.

Podolak M. and Prialnik D. (2000) Conditions for the production of liquid water in comet nuclei. In Bioastronomy '99: A New Era in Bioastronomy (G. A. Lemarchand and K. J. Meech, eds.), pp. 231-234. Sheridan, Chelsea.

Prialnik D. (1992) Crystallization, sublimation, and gas release in the interior of a porous comet nucleus. Astrophys. J., 388, 196-202.
Prialnik D. (1999) Modelling gas and dust release from Comet Hale-Bopp. Earth Moon Planets, 77, 223-230.

Prialnik D. (2002) Modeling the comet nucleus interior; application to Comet C/1995 O1 Hale-Bopp. Earth Moon Planets, 89, 27-52.

Prialnik D. and Bar-Nun (1987) On the evolution and activity of cometary nuclei. Astrophys. J., 313, 893-905.

Prialnik D. and Bar-Nun (1988) Formation of a permanent dust mantle and its effect on cometary activity. Icarus, 74, 272283

Prialnik D. and Bar-Nun (1990) Gas release in comet nuclei. Astrophys. J., 363, 274-282.

Prialnik D. and Bar-Nun (1992) Crystallization of amorphous ice as the cause of Comet P/Halley's outburst at 14 AU. Astron. Astrophys., 258, L9-L12.

Prialnik D. and Mekler Y. (1991) The formation of an ice crust below the dust mantle of a cometary nucleus. Astrophys. J., 366, 318-323.

Prialnik D. and Podolak M. (1995) Radioactive heating of porous cometary nuclei. Icarus, 117, 420-430.

Prialnik D., Egozi U., Bar-Nun A., Podolak M., and Greenzweig Y. (1993) On pore size and fracture in gas - laden comet nuclei. Icarus, 106, 499-507.

Prialnik D., Brosch N., and Ianovici D. (1995) Modelling the activity of 2060 Chiron. Mon. Not. R. Astron. Soc., 276, 11481154.

Rayleigh Lord (1892) On the influence of obstacles arranged in rectangular order upon the properties of a medium. Phil. Mag., 56, 481-502.

Reach W. T., Sykes M. V., Lien D. J., and Davies J. K. (2000) The formation of Encke meteoroids and dust trail. Icarus, 148, 80-89.

Rickman H. (1989) The nucleus of Comet Halley: Surface structure, mean density, gas and dust production. Adv. Space Res., 9, 59-71.

Rickman H., Fernández J. A., and Gustafson B. Å. S. (1990) Formation of stable dust mantles on short-period comet nuclei. Astron. Astrophys., 237, 524-535.

Ross R. G. and Kargel J. S. (1998) Thermal conductivity of solar system ices with special reference to Martian polar caps. In Solar System Ices (B. Schmitt et al., eds.), pp. 33-62. Kluwer, Dordrecht.

Schmitt B., Espinasse S., Grin R. J. A., Greenberg J. M., and Klinger J. (1989) Laboratory studies of cometary ice analogues. In Proceedings of an International Workshop on Physics and Mechanics of Cometary Materials (J. Hunt and T. D. Guyeme, eds.), pp. 65-69. ESA SP-302, Noordwijk, The Netherlands.

Schmitt B., Espinasse S., and Klinger J. (1991) A possible mechanism for outbursts of Comet P/Halley at large heliocentric distances (abstract). In Abstracts for the 54th Annual Meeting of the Meteoritical Society, p. 208. LPI Contribution No. 766, Lunar and Planetary Institute, Houston.

Seiferlin K., Koemle N. I., Kargl G., and Spohn T. (1996) Line heat-source measurements of the thermal conductivity of porous ice, ice and mineral powders under space conditions. Planet. Space Sci., 44, 691-704.

Sekanina Z. (1979) Fan-shaped coma, orientation of rotation axis, and surface structure of a cometary nucleus. I - Test of a model on four comets. Icarus, 37, 420-442.

Sekanina Z. (1984) Disappearance and disintegration of comets. Icarus, 58, 81-100. 
Senay M. C. and Jewitt D. (1994) Coma formation driven by carbon monoxide release from Comet P/Schwassmann-Wachmann 1. Nature, 371, 229-231.

Shoshany Y., Heifetz E., Prialnik D., and Podolak M. (1997) A model for the changing pore structure and dust grain size distribution in a porous comet nucleus. Icarus, 126, 342-350.

Shoshany Y., Podolak M., and Prialnik D. (1999) A Monte-Carlo model for the flow of dust in a porous comet nucleus. Icarus, 137, 348-354.

Shoshany Y., Prialnik D., and Podolak M. (2002) Monte-Carlo modeling of the thermal conductivity of cometary ice. Icarus, 157, 219-227.

Shul'man L. M. (1972) The evolution of cometary nuclei. In The Motion, Evolution of Orbits, and Origin of Comets (G. A. Chebotarev et al., eds.), p. 271. IAU Symposium No. 45, Reidel, Dordrecht.

Sirono S. and Greenberg J. M. (2000) Do cometesimal collisions lead to bound rubble piles or to aggregates held together by gravity? Icarus, 145, 230-238.

Sirono S. and Yamamoto T. (1997) Thermal conductivity of granular material relevant to the thermal evolution of comet nuclei. Planet. Space Sci., 45, 827-834.

Skorov Yu. V. and Rickman H. (1995) A kinetic model of gas flow in a porous cometary mantle. Planet. Space Sci., 43, 1587 1594.

Skorov Yu. V. and Rickman H. (1998) Simulation of gas flow in a cometary Knudsen layer. Planet. Space Sci., 46, 975-996.

Skorov Yu. V. and Rickman H. (1999) Gas flow and dust acceleration in a cometary Knudsen layer. Planet. Space Sci., 47, 935-949.

Skorov Yu. V., Kömle N. I., Keller H. U., Kargl G., and Markiewicz W. J. (2001) A model of heat and mass transfer in a porous cometary nucleus based on a kinetic treatment of mass flow. Icarus, 153, 180-196.

Smoluchowski R. (1981) Amorphous ice and the behavior of cometary nuclei. Astrophys. J. Lett., 244, L31-L36.

Smoluchowski R. (1982) Heat transport in porous cometary nuclei. Proc. Lunar Planet Sci. Conf. 13th, in J. Geophys. Res., 87, A422-A424.

Squires R. E. and Beard D. B. (1961) Physical and orbital behavior of comets. Astrophys. J., 133, 657-667.

Squyres S. W., McKay C. P., and Reynolds R. T. (1985) Temperatures within comet nuclei. J. Geophys. Res., 90, 12381-12392.
Spohn T., Seiferlin K., and Benkhoff J. (1989) Thermal conductivities and diffusivities of porous ice samples at low pressures and temperatures and possible modes of heat transfer in near surface layers of comets. In Proceedings of an International Workshop on Physics and Mechanics of Cometary Materials (J. Hunt and T. D. Guyeme, eds.), pp. 77-81. ESA SP-302, Noordwijk, The Netherlands.

Srinivasan G., Goswami J. N., and Bhandari N. (1999) ${ }^{26} \mathrm{Al}$ in eucrite Piplia Kalan: Plausible heat source and formation chronology. Science, 284, 1348-1350.

Stauffer D. and Aharony A. (1994) Introduction to Percolation Theory, 2nd edition. Taylor and Francis, Bristol. $181 \mathrm{pp}$.

Steiner G. and Kömle N. I. (1991) A model of the thermal conductivity of porous water ice at low gas pressures. Planet. Space Sci., 39, 507-513.

Steiner G. and Kömle N. I. (1993) Evolution of a porous $\mathrm{H}_{2} \mathrm{O}-$ $\mathrm{CO}_{2}$-ice sample in response to irradiation. J. Geophys. Res., 98, 9065-9073.

Tancredi G., Rickman H., and Greenberg J. M. (1994) Thermal chemistry of a cometary nuclei. I. The Jupiter family case. Astron. Astrophys., 286, 659-682.

Thomas P. J., Chyba C. F., and McKay C. P., eds. (1997) Comets and the Origin and Evolution of Life. Springer-Verlag, New York. 296 pp.

Wallis M. K. (1980) Radiogenic heating of primordial comet interiors. Nature, 284, 431-433.

Weidenschilling S. J. (1997) The origin of comets in the solar nebula: A unified model. Icarus, 127, 290-306.

Weissman P. R. (1991) Why did Halley hiccup? Nature, 353, 793794.

Weissman P. R. and Kieffer H. H. (1981) Thermal modeling of cometary nuclei. Icarus, 47, 302-311.

Weissman P. R. and Kieffer H. H. (1984) An improved thermal model for cometary nuclei. J. Geophys. Res. Suppl., 89, 358364.

West R. M., Hainaut O., and Smette A. (1991) Post-perihelion observations of P/Halley. III - An outburst at R = 14.3 AU. Astron. Astrophys., 246, L77-L80.

Whipple F. L. and Stefanik R. P. (1966) On the physics and splitting of cometary nuclei. Mem. R. Soc. Liege (Ser. 5), 12, 33-52.

Yabushita S. (1993) Thermal evolution of cometary nuclei by radioactive heating and possible formation of organic chemicals. Mon. Not. R. Astron. Soc., 260, 819-825. 
